

Qu, H., Liu, G., Zhang, L., Imran, M. A. and Wen, S. (2021) Low-dimensional subspace estimation of continuous-doppler-spread channel in OTFS systems. IEEE Transactions on Communications, (doi: 10.1109/TCOMM.2021.3072744)

There may be differences between this version and the published version. You are advised to consult the publisher's version if you wish to cite from it.

http://eprints.gla.ac.uk/238160/

Deposited on: 16 April 2021

Enlighten - Research publications by members of the University of Glasgow http://eprints.gla.ac.uk 


\title{
Low-Dimensional Subspace Estimation of Continuous-Doppler-Spread Channel in OTFS Systems
}

\author{
Huiyang Qu, Guanghui Liu, Senior Member, IEEE, Lei Zhang, Senior Member, IEEE, \\ Muhammad Ali Imran, Senior Member, IEEE, and Shan Wen, Student Member, IEEE
}

\begin{abstract}
Orthogonal time frequency space (OTFS) has shown to be a promising modulation technology that achieves the robust wireless transmission in high-mobility environments. The high mobility incurred Doppler effect in OTFS system, is represented as a continuous and relatively large band in the Doppler frequency. It yields the equivalent channel responses (ECRs) in the system change significantly within one symbol block, posing a challenge to channel estimation (CE) or tracking. In order to tackle this issue, in this paper, a set of transformdomain basis functions is designed to span a low-dimensional subspace for modeling the OTFS channel. Then, the CE can be performed by estimating a few projection coefficients of ECRs in the developed subspace, with training pilots. According to the individual transmission characteristic of OTFS signal, we propose a corner-inserted pilot pattern, which targets the low pilot overhead and satisfactory CE performance. Moreover, an OTFS signal detector, leveraging the time-domain channel equalization, linear-complexity interference cancellation and delay-Doppler domain maximal ratio combining detection, is developed to retrieve the transmitted data symbols. The simulations show the precisely estimated ECRs enable the detector to ideally demodulate 256-ary quadrature amplitude modulation signaling, under a velocity of $550 \mathrm{~km} / \mathrm{h}$ at $5.9 \mathrm{GHz}$ carrier frequency.
\end{abstract}

Index Terms-Orthogonal time frequency space (OTFS), channel estimation, channel equalization, high mobility, 256QAM

\section{INTRODUCTION}

Future wireless communications are envisioned to support diverse use cases and requirements. Identified as one of the most challenging communication scenarios, reliable communications in high-mobility environments, such as vehicles to everything networks (V2X) [1], [2], high-speed trains [3], [4], and unmanned aerial vehicles [5], [6], have attracted much attention from both academia and industry. Unlike other use cases, the high-mobility scenarios feature severe dispersion of radio channel in the time-frequency (TF) domain, which poses a performance bottleneck to the traditional multi-carrier

This work was supported in part by the National Natural Science Foundation of China under Grants 62071097, U20A20184, and in part by the 111 Project under Grant B17008. (Corresponding author: Guanghui Liu).

Huiyang Qu, Guanghui Liu and Shan Wen are with the School of Information and Communication Engineering, University of Electronic Science and Technology of China, Chengdu 611731, China (e-mail: guanghuiliu@uestc.edu.cn; \{hyqu, shanwen\}@std.uestc.edu.cn).

Lei Zhang and Muhammad Ali Imran are with the James Watt School of Engineering, University of Glasgow, Glasgow, G12 8QQ, U.K. (e-mail: \{Lei.Zhang, Muhammad.Imran\}@glasgow.ac.uk). modulation system, such as orthogonal frequency division multiplexing (OFDM) [7]-[9] or its varieties [10]-[12].

Recently, the so-called orthogonal time frequency space (OTFS) modulation technique, has been developed to improve the wireless transmission performance in high mobilities [13]-[15]. The key point of OTFS is to model the radio channel in the delay-Doppler (DD) domain [16]. Based on this representation, OTFS maps data symbols on grid points in a DD plane and employs inverse symplectic finite Fourier transform (ISFFT) [17] to spread them over the whole considered TF grids. Then, the TF-domain data symbols are modulated by a multi-carrier scheme, e.g., OFDM [18]. The OTFS signaling enables the transmitted data symbols to be equally impacted from the doubly-dispersive channel, meanwhile the full diversity from the channel can be reaped by the efficient receiver's equalization [19]-[22].

Accurate channel estimation (CE) is a prerequisite for the robust demodulation in the OTFS receiver. In some highmobility environments, such as high-speed train running in a clear and open signal-transmission space [23], the channel scattering components are limited and the channel responses may appear as a few impulses in the Doppler frequency [24]. In such a limited-Doppler-shift channel (LDSC), the existing CE schemes [25]-[28] are efficient to acquire the sparse DDdomain channel gains. For instance, in [25], the base station transmits a single impulse (SI) signal as pilot with a proper guard to estimate the DD-domain channel gains by means of a threshold method. The SI based CE was extended to MIMO-OTFS system in [26]. An alternative approach is the pseudo-random pilot based CE [27], which reconstructs the DD channel by utilizing the estimated parameters, e.g., the delay/Doppler shifts and the corresponding gains. Moreover, the compressive sensing (CS) technique has also been used to estimate the DD channel in [28].

To the best of our knowledge, the existing schemes are designed only to estimate LDSCs. However, a more general communication environment involves a large number of scattering objects, like in V2X. The scattering-abundant channel is treated as continuous-Doppler-spread channel (CDSC) [29], in which the Doppler frequency components of each transmission path are infinite and the Doppler spectrum is continuous in frequency rather than a set of discrete frequency elements. The motivations of this paper originate from the case that 
OTFS signal undergoes the CDSC 1 Specifically,

1) Focusing on high-speed mobile scenarios, our study indicates that the equivalent channel responses (ECRs) in OTFS system can change quickly within one symbol block. However, the existing CE algorithms, developed for the LSDC, are insufficient to accurately track the inside-one-symbol variations of ECRs. Once ignoring the variations, severe demodulation error occurs.

2) When the variations can not be ignored, the number of unknown ECRs is much larger than the observations (pilot symbols), which means the CE becomes an undertermined estimation problem. Thus, how to precisely estimate the unknowns with the limited number of training pilots is another concern of this paper.

To solve this problem, a low-dimensional subspace based $\mathrm{CE}$ scheme is proposed to track the variable ECRs. The designed channel estimator is capable of achieving the whole (not just a part of) ECRs, and preserving a low pilot overhead. Benefitting from the accurate channel estimates, the develope$\mathrm{d}$ detector can ideally retrieve the OTFS data symbols in the CDSC. Our contributions are summarized as follows.

1) By investigating the characteristics of OTFS channels, a low-dimensional subspace is constructed to characterize the variations of the ECRs. The subspace is expanded by a series of transform-domain basis functions. Then, the ECRs are precisely modeled in the subspace, by a sum of the projection coefficients and the basis functions.

2) The subspace channel modeling significantly reduces the number of unknowns, and meanwhile simplifies the OTFS CE as acquiring the projection coefficients of ECRs, by solving an overdertermined estimation problem. The linear minimum mean square error (LMMSE) based channel estimator employs the training pilots, which are inserted in a form of the proposed cornerinserted pilot pattern (CIPP), to obtain the projection coefficients.

3) Combining with the channel estimator, an iterative OTFS symbol detector, which involves least squares QR decomposition (LSQR) channel equalization, linearcomplexity interference cancellation and maximal ratio combing (MRC), is developed for OTFS system. The simulation results demonstrate the superiority of OTFS receiver adopting the proposed $\mathrm{CE}$ and equalization schemes. For example, when the maximum Doppler frequency normalized to the subcarrier spacing $\tilde{f}_{d}=$ $20 \%$, corresponding to a velocity of $550 \mathrm{~km} / \mathrm{h}$ at 5.9 $\mathrm{GHz}$ carrier frequency, the proposed receiver can robustly demodulate 256-ary quadrature amplitude modulation (QAM) symbols at a suitable signal-to-noise ratio (SNR) regime. Moreover, from this paper, an op-

\footnotetext{
${ }^{1}$ The CDSC can be treated as a kind of definition or expression of the channels that have continuous Doppler spectrum $S(f)$ in the frequency $f$. Note that the channel Doppler spectrum $S(f)$ is a result of Fourier transform to the channel correlation function, i.e., $S(f)=\mathrm{FT}(r(\tau))$, where $r(\tau)=\mathbb{E}\left\{h(t) h^{*}(t-\tau)\right\}$. In the scattering-abundant scenarios, the infinite electromagnetic waves arrive at the reviver, yielding a continuous Doppler spread in the frequency.
}

timal OTFS transmission scheme considering the data transmission efficiency, pilot power and demodulation performance, can be achieved for the diverse mobile scenarios and traffic loads.

The remainder of this paper is organized as follows. The OTFS signaling and the ECRs are formulated in Section II. In Section III, the low-dimensional subspace is developed to model the OTFS channel and transform the CE into projection coefficient estimation. The proposed pilot pattern and training pilot based coefficient estimation are presented in Section IV. In Section V, the LSQR channel equalization aided MRC detector is detailed. Finally, simulation results are presented in Section VI and conclusions are given in Section VII.

Notations: In this paper, we use upper (lower) boldface letters denote matrices (column vectors). The element in the $v$-th row and $l$-th column of matrix $\mathbf{Y}$ is denoted as $Y(l, v)$. The $m$-th row and $n$-th column of matrix $\mathbf{X}$ are denoted as $\mathbf{X}(m,:)$ and $\mathbf{X}(:, n)$, respectively. We use $(\cdot)^{-1},(\cdot)^{T}$ and $(\cdot)^{H}$ to represent inverse, transpose and conjugate transpose, respectively. $\mathbf{R}_{\mathbf{c}}=$ blkdiag $\left\{\mathbf{R}_{0}, \mathbf{R}_{1}, \cdots, \mathbf{R}_{N-1}\right\}$ is a block diagonal matrix with the matrices $\mathbf{R}_{0}, \mathbf{R}_{1}, \cdots, \mathbf{R}_{N-1}$ on its main diagonal. Circ $(\cdot)$ represents cyclic shift operation on row vector $\mathbf{x} \in \mathbb{C}^{1 \times N}$, and $\operatorname{Circ}(\mathbf{x}, a)=$ $[x(N-a), \cdots, x(N-1), x(0), \cdots, x(N-a-1)]$. Operators vec $(\cdot), \odot, \otimes,(\cdot)_{N}\lceil\cdot\rceil,\|\cdot\|, \operatorname{Tr}(\cdot)$ and $\mathbb{E}(\cdot)$ are denoted as the vectorizing of a matrix, Hadamard product, kronecker product, modulo-N operation, ceil operator, Frobenius norm, trace operation and expectation operation, respectively. $\mathbb{C}$ stands for the set of complex numbers. $\mathbf{I}_{M}$ denotes the $M \times M$ identity matrix. Finally, we denote the zeroth order Bessel function of the first kind as $J_{0}(\cdot)$.

\section{OTFS TRANSMISSION OVER CDSC}

In this section, we first formulate the input-output relation of OTFS signal transmission over CDSC. Then, the ECRs are derived by transforming the channel impluse responses (CIRs) from time-delay (TD) domain to DD domain. The transformation provides us some insights into the OTFS CE in high mobilities.

\section{A. OTFS Signaling}

The discrete-time OTFS transmission architecture is illustrated in Fig. 1. The modulated data symbols and the pilot sequences are arranged into a signal matrix $\mathbf{X} \in \mathbb{C}^{M \times N}$, where $M$ and $N$ are the numbers of resource grids along the delay and Doppler dimensions, respectively. The ISFFT converts $\mathbf{X}$ from the DD domain to the TF domain, i.e., $\mathbf{D}=\mathbf{F}_{M} \mathbf{X F}_{N}^{H}, \mathbf{D} \in \mathbb{C}^{M \times N}$, where $\mathbf{F}_{M} \in \mathbb{C}^{M \times M}$ and $\mathbf{F}_{N} \in \mathbb{C}^{N \times N}$ are the fast Fourier transform (FFT) matrices [17]. Subsequently, the time-domain signal block is generated by $\mathbf{S}=\mathbf{F}_{M}^{H} \mathbf{D}, \mathbf{S} \in \mathbb{C}^{M \times N}$. By column-wise reading the entries in $\mathbf{S}$, the data samples within one OTFS transmission block are: $\mathbf{s}=\operatorname{vec}(\mathbf{S}), \mathbf{s} \in \mathbb{C}^{M M \times 1}$. A cyclic prefix (CP) with length $M_{\mathrm{cp}}$ is inserted at the beginning of $\mathbf{s}$ [19] to avoid the inter-block interference. The discrete-time OTFS sequences go through the doubly selective channel, of which 




Fig. 1. The baseband transmission architecture of OTFS system.

the impulse response is defined as $h\left[n, l^{\prime}\right], l^{\prime} \in[0, L-1]$, where the maximum channel delay spread $L \leq M_{\mathrm{cp}}$.

At the receiver side, after removing $\mathrm{CP}$, the received timedomain signal block $\mathbf{R} \in \mathbb{C}^{M \times N}$ is transformed to the TF domain by means of FFT in OFDM demodulator, which is expressed as $\mathbf{D}^{\prime}=\mathbf{F}_{M} \mathbf{R}, \mathbf{D}^{\prime} \in \mathbb{C}^{M \times N}$. Then, the symplectic finite Fourier transform (SFFT) converts $\mathbf{D}^{\prime}$ back into the DD domain, according to $\mathbf{Y}=\mathbf{F}_{M}^{H} \mathbf{D}^{\prime} \mathbf{F}_{N}, \mathbf{Y} \in \mathbb{C}^{M \times N}$.

Proposition 1: Aided by the existing works from [28], [30][32], the received signal $Y(l, v), l \in[0, M-1], v \in$ $[0, N-1]$, which corresponds to the $(l, v)$-th element of $\mathbf{Y}$, is derived as

$$
Y(l, v)=\sum_{v^{\prime}=0}^{N-1} \sum_{l^{\prime}=0}^{M-1} \Omega\left\{l, l^{\prime}, v^{\prime}\right\} X\left(\left(l-l^{\prime}\right)_{M},\left(v-v^{\prime}\right)_{N}\right)
$$

In 11), $W(l, v)$ represents the additive white Gaussian noise with zero mean and variance $\sigma^{2} ; X\left(\left(l-l^{\prime}\right)_{M},\left(v-v^{\prime}\right)_{N}\right)$ denotes the $\left(\left(l-l^{\prime}\right)_{M},\left(v-v^{\prime}\right)_{N}\right)$-th entry of $\mathbf{X}$; $\Omega\left\{l, l^{\prime}, v^{\prime}\right\}, l \in[0, M-1], l^{\prime} \in[0, L-1], v^{\prime} \in[0, N-1]$ is the ECR in OTFS system

$$
\Omega\left\{l, l^{\prime}, v^{\prime}\right\}=\frac{1}{N} \sum_{i=0}^{N-1} e^{\frac{-j 2 \pi i v^{\prime}}{N}} h\left[i M+M_{\mathrm{cp}}+l, l^{\prime}\right] .
$$

Note that $\Omega\left\{l, l^{\prime}, v^{\prime}\right\}$ is the function of three variables: $l$, $l^{\prime}$ and $v^{\prime}$, which represent the delay-direction index in DD plane, channel delay-sampling index and channel Dopplersampling index, respectively. The $l$-th delay-direction index corresponds to the symbol $Y(l, v)$ that located in the $l$-th delay grid, and it varies from 0 to $M-1$. As the maximum channel delay spread is $L$, the channel delay-sampling index $l^{\prime}$ changes from 0 to $L-1$. The $v^{\prime}$-th channel Doppler sampling is associated with the $i$-th OFDM symbol in (2), where $v^{\prime}$ and $i$ change from 0 to $N-1$. To distinguish $l$ from $l^{\prime}$, we call $l$ as group index hereafter.


Fig. 2. The illustration of 2-D convolution in 11, where the maximum channel delay spread $L=4$.

Fig. 2 is presented to demonstrate the 2-D convolution in
(1), where a single OTFS impulse is allocated in the transmitted DD plane. From Fig. 2, the transmitted OTFS signal is circularly shifted by the channel delay/Doppler spreads and scaled by the ECR $\Omega\left\{l, l^{\prime}, v^{\prime}\right\}, l \in[0, M-1], l^{\prime} \in$ $[0, L-1], v^{\prime} \in[0, N-1]$. In particular, when Doppler spread is zero, the channel is time-invariant, of which the CIR at the $l^{\prime}$-th path can be denoted as $a_{l^{\prime}}$. In this case, 2 is transformed to $\Omega\left\{l, l^{\prime}, v^{\prime}\right\}=a_{l^{\prime}} \delta\left(v^{\prime}\right) u(l)$, demonstrating the ECR is constant at the $l^{\prime}$-th delay spread and zeroth Doppler frequency. Thus, in (1), the transmitted OTFS symbols will only be circularly shifted by the delay spread and scaled by $a_{l^{\prime}}$. When the delay spread is zero, we can derive the similar conclusion.

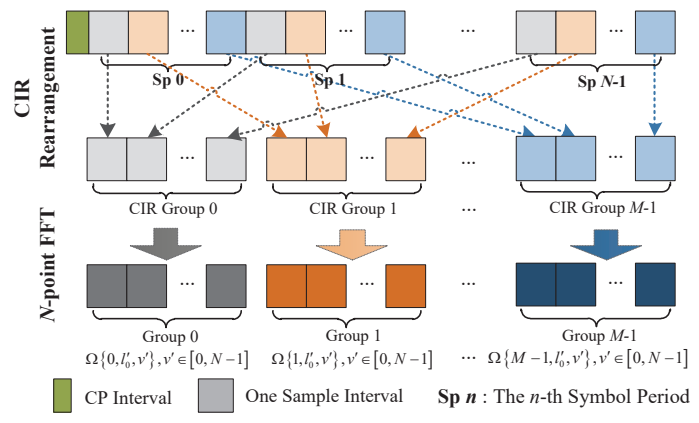

Fig. 3. The conversion process between the CIRs and ECRs in OTFS system.

Fig. 3 demonstrates the transformation between the CIRs and ECRs in OTFS system. The TD-domain CIR samples of the $l_{0}^{\prime}$-th path ${ }^{2}$, within one OTFS transmission block, are rearranged into $M$ groups, each of which includes $N$ points. The CIR samples in the $l$-th group $(l=0, \cdots, M-1)$, i.e., $h\left[i M+M_{\mathrm{cp}}+l, l_{0}^{\prime}\right], i \in[0, N-1]$, are converted to $\Omega\left\{l, l_{0}^{\prime}, v^{\prime}\right\}, v^{\prime} \in[0, N-1]$ through $N$-point FFT as 22 .

\section{B. Analysis of $\Omega\left\{l, l^{\prime}, v^{\prime}\right\}$ over CDSC}

The CDSC is based on the statistical channel modeling method, which has been widely used in the practical wireless communications. According to the existing measurement campaigns in the high-mobility wireless communications [29], [33], there can be abundant scatters/reflectors around the mobile terminals [34]. The scattered/reflected electromagnetic waves uniformly distributing around the reviver (which has isotropic antenna), results in a continuous U-shaped Doppler

\footnotetext{
${ }^{2}$ Here, we take one channel path, with delay spread $l_{0}^{\prime}$, to demonstrate the transformation.
} 
spread/spectrum in the frequency, directly corresponding to that in the classical Clarke channel [35]. As shown in Fig. 4 . the Clarke channel occupies a continuous band in the Doppler frequency, i.e., $\left[-f_{d}, f_{d}\right]$, where $f_{d}$ is the maximum Doppler frequency. As a typical case of CDSC, the classical Clarke channel model [35] is selected in this paper to help us analyze $\Omega\left\{l, l^{\prime}, v^{\prime}\right\}$. Note that our analysis is not only applicable to Clarke channel, but also can be valid in other cases of CDSC.

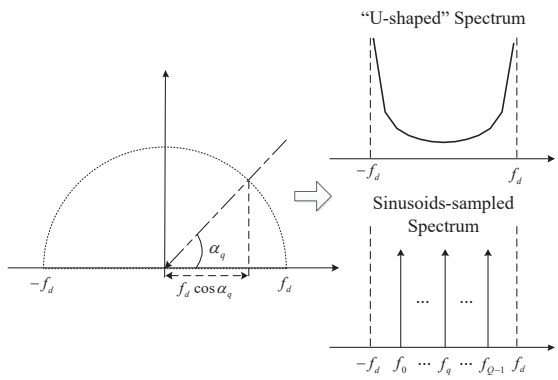

Fig. 4. Clarke channel spectrum in the Doppler frequency, 1) "U-shaped" spectrum; 2) "Sinusoids-sampled/approximated" spectrum.

In general, a series of sinusoids with frequencies are commonly adopted to approximate the continuous U-shaped spectrum. Thus, the time-variant CIR of the $l^{\prime}$-th path can be modeled as

$h\left[n, l^{\prime}\right]=\sum_{q=0}^{Q-1} \alpha_{l^{\prime}, q} e^{j\left(2 \pi f_{l^{\prime}, q} n T_{s}+\theta_{l^{\prime}, q}\right)} \triangleq \sum_{q=0}^{Q-1} \tilde{\alpha}_{l^{\prime}, q} e^{j 2 \pi f_{l^{\prime}, q} n T_{s}}$,

where $\tilde{\alpha}_{l^{\prime}, q}=\alpha_{l^{\prime}, q} e^{j \theta_{l^{\prime}, q}, T_{s}}$ is the system sampling period and $Q$ denotes the number of sinusoids (or approximated Doppler shift components) of the transmission path ${ }^{3}$ Parameters $\theta_{l^{\prime}, q}, \alpha_{l^{\prime}, q}$ and $f_{l^{\prime}, q}$ represent the $q$-th initial phase, complex gain and Doppler shift associated with the $l^{\prime}$-th path, respectively. Substituting (3) into (2), we have

$$
\Omega\left(l, l^{\prime}, v^{\prime}\right)=\sum_{q=0}^{Q-1} \tilde{\alpha}_{l^{\prime}, q} e^{j 2 \pi \bar{f}_{l^{\prime}, q}\left(M_{\mathrm{cp}}+l\right) / M N} \Xi_{N}\left(v^{\prime}-\bar{f}_{l^{\prime}, q}\right),
$$

where

$$
\Xi_{N}(x)=\frac{1}{N} \sum_{i=0}^{N-1} e^{\frac{-j 2 \pi i x}{N}}=\frac{\sin (\pi x)}{N \sin \left(\pi \frac{x}{N}\right)} e^{-j \pi \frac{x(N-1)}{N}} .
$$

In (4), $\bar{f}_{l^{\prime}, q}=f_{l^{\prime}, q} N M T_{s} \triangleq f_{l^{\prime}, q} T_{D}$ denotes the sampled channel Doppler shift, where $T_{D}=N M T_{s}=\Delta f / N$ is the sampling resolution of OTFS system in the Doppler dimension.

According to (4), the changes of $\Omega\left(l, l^{\prime}, v^{\prime}\right)$ related to the group index $l$, are reflected in the phase accumulation $e^{j 2 \pi \bar{f}_{l^{\prime}, q}\left(M_{\mathrm{cp}}+l\right) / M N}$. In some low-speed scenarios, the channel Doppler bandwidth $2 f_{d}$ is much lower than the signal bandwidth. Thus, $e^{j 2 \pi \bar{f}_{l^{\prime}, q}\left(M_{\mathrm{cp}}+l\right) / M N}$ can be regarded as constant for $l \in[0, M-1]$, since the values of $\bar{f}_{l^{\prime}, q}$ are

\footnotetext{
${ }^{3}$ The number of Doppler-shifted sinusoids, $Q$, must be large enough to approximate the amplitude of the fading channel with a Rayleigh distribution. In general, it has been known that $Q \geq 8$ or 16 is satisfied [36].
}

considerably small. As a result, for each group $l, \Omega\left\{l, l^{\prime}, v^{\prime}\right\}$ is almost identical in Fig. 3. However, in high-mibility environments, the values of $\bar{f}_{l^{\prime}, q}$ are significant due to the larger channel Doppler bandwidth. In this case, $e^{j 2 \pi \bar{f}_{l^{\prime}, q}\left(M_{\mathrm{cp}}+l\right) / M N}$ is variable, and $\Omega\left\{l, l^{\prime}, v^{\prime}\right\}$ can change obviously between groups.

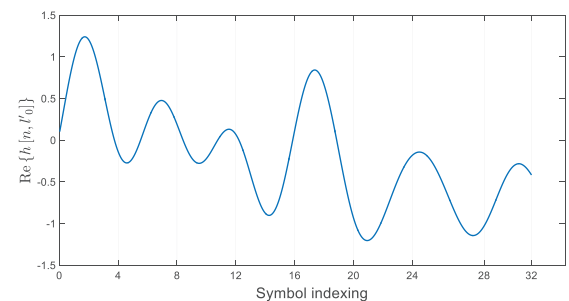

(a) The real part of the time-domain CIR curve (corresponding to the $l_{0}^{\prime}$-th path).



(b) The real parts of the ECRs of the $l_{0}^{\prime}$-th path.

Fig. 5. The TD-domain CIRs $h\left[n, l_{0}^{\prime}\right]$ and ECRs $\Omega\left\{l, l_{0}^{\prime}, v^{\prime}\right\}$, where the OTFS transmission block $M N=32 \times 32$. The maximum channel Doppler frequency normalized to the subcarrier spacing $\tilde{f}_{d}$ is $20 \%$, corresponding to a velocity of $550 \mathrm{~km} / \mathrm{h}$.

To illustrate this, we plot $h\left[n, l^{\prime}\right]$ and $\Omega\left\{l, l^{\prime}, v^{\prime}\right\}$ of a certain path with the delay spread $l_{0}^{\prime}$ in Fig. 5, where $\tilde{f}_{d} \triangleq f_{d} / \Delta f=20 \%$. It can be seen from Fig. 5(a) that the TDdomain CIR varies significantly within $N$ OFDM symbols (in Fig. 5. $N=32$ ). Fig. 5(b) demonstrates the real parts of $\Omega\left\{l, l_{0}^{\prime}, v^{\prime}\right\}$. We can see that, at some Doppler indices $v^{\prime}, \Omega\left\{l, l_{0}^{\prime}, v^{\prime}\right\}$ changes rapidly with respect to the group index $l, l \in[0, M-1]$ (in Fig. 5. $M=32$ ). As a result, the intra-group variations of $\Omega\left\{l, l^{\prime}, v^{\prime}\right\}$ cannot be ignored in high mobilities.

The CE task is to acquire $\Omega\left\{l, l^{\prime}, v^{\prime}\right\}$ in 11 , including $M \times L \times N$ unknown gains. However, it might be hard to directly estimate these unknowns since the number of observation data $Y(l, v)$ is $M \times N$. In other words, it is an underdetermined estimation problem. Thus, an effective modeling scheme has to be taken to reduce the dimension of channel responses, which necessitates the low-dimensional subspace based channel modeling in Section III.

Remark 1: There is a natural and effective CE method, i.e., transmitting an SI as pilot signal to experience the channel [25]. In this way, we can estimate one group of $\Omega\left(l, l^{\prime}, v^{\prime}\right)$ from the observed signal in the DD plane, and further reconstruct $\tilde{\alpha}_{l^{\prime}, q}$ and $\bar{f}_{l^{\prime}, q}$ to predict the responses in other groups. The problem is that reconstructing $\tilde{\alpha}_{l^{\prime}, q}$ and $\bar{f}_{l^{\prime}, q}$ in (4) is not easy, since the Doppler-sampling resolution $T_{D}$ may 
not sufficient to distinguish each physical channel Doppler shift. As a result, the prediction accuracy of $\Omega\left(l, l^{\prime}, v^{\prime}\right)$ in other groups would not be reliable over high-Doppler-spread channels.

Remark 2: A straightforward strategy for compensating the above shortage is to increase the value of $N$ [28], yielding a higher Doppler-sampling resolution. In particular, when $N \rightarrow$ $\infty$, each $\bar{f}_{l^{\prime}, q}$ can be ideally quantized and 44 is transformed as

$$
\Omega\left\{l, l^{\prime}, v^{\prime}\right\} \stackrel{N \rightarrow \infty}{=} e^{\frac{j 2 \pi\left(M_{\mathrm{cp}}+l\right) v^{\prime}}{N M}} \Omega\left\{0, l^{\prime}, v^{\prime}\right\} .
$$

Hence, from (5), the prediction for all groups becomes much easier. Unfortunately, in practical OTFS system, $N$ cannot be considered so large due to the limited computational capability and low-latency decoding requirement. When $N$ is relatively small, it will introduce a considerably large formulation error in (5) and thus deteriorate the CE accuracy.

\section{Low-Dimensional SubSPACE BASED CHANNEL MODELING}

The low-dimensional subspace based channel modeling is proposed in this section, for reducing the dimension of $\Omega\left\{l, l^{\prime}, v^{\prime}\right\}$. The developed channel modeling scheme enables to simplify the OTFS CE as acquiring the projection coefficients of ECRs, by solving an overdertermined estimation problem.

\section{A. Low-Dimensional Subspace Construction}

A set of transform-domain basis functions is designed to construct a $K$-dimensional $(K \ll M)$ subspace for characterizing the variations of $\Omega\left\{l, l^{\prime}, v^{\prime}\right\}$. Specifically, $\Omega\left\{l, l^{\prime}, v^{\prime}\right\}$ is approximated in this subspace, i.e.,

$$
\Omega\left\{l, l^{\prime}, v^{\prime}\right\}=\sum_{k=1}^{K} c_{k, l^{\prime}} A\left(l, k, v^{\prime}\right)+e_{\mathrm{D}}\left\{l, l^{\prime}, v^{\prime}\right\}
$$

where $e_{\mathrm{D}}\left\{l, l^{\prime}, v^{\prime}\right\}$ is the residual error and $c_{k, l^{\prime}}$ denotes the $k$-th projection coefficient of the $l^{\prime}$-th path. In $(6), A\left(l, k, v^{\prime}\right)$ is the $k$-th transform-domain basis function, describing the variable $l$ at the $v^{\prime}$-th Doppler frequency. From (6), the variable $l$ is separated from $\Omega\left\{l, l^{\prime}, v^{\prime}\right\}$ and then incorporated into $A\left(l, k, v^{\prime}\right)$. Since $A\left(l, k, v^{\prime}\right)$ is a known function of $l$, the number of unknowns corresponding to the $l^{\prime}$-th path is reduced from $M N$ to $K$. Note that the value of $K$ is crucial to the accuracy of subspace channel modeling and how to select $K$ will be discussed in detail in Sections III-B and III-C. Recalling (2), for the $l^{\prime}$-th path, the variations of $\Omega\left\{l, l^{\prime}, v^{\prime}\right\}$ in the group and Doppler directions, directly relates to the time variation of $h\left[i M+M_{\mathrm{cp}}+l, l^{\prime}\right]$. Accordingly, we propose to construct a time-domain orthogonal space V1 to model the time variations of $h\left[i M+M_{\mathrm{cp}}+l, l^{\prime}\right]$. Then, by transforming the basis functions in V1 from the time domain to transform domain, we can further achieve the subspace V2, to model $\Omega\left\{l, l^{\prime}, v^{\prime}\right\}$ as $(6)$.

Specifically, the time-domain orthogonal subspace V1 is expanded by a series of time-domain basis functions, i.e.,

$$
\mathrm{V} 1: \operatorname{span}\left\{\mathbf{b}_{1}, \mathbf{b}_{2}, \cdots, \mathbf{b}_{K}\right\},
$$

where $\mathbf{b}_{k}$ denotes the $k$-th basis function, $\mathbf{b}_{k}=\left[b(0, k), b(1, k), \cdots, b\left(M N+M_{\mathrm{cp}}-1, k\right)\right]^{T} \in$ $\mathbb{C}^{\left(M N+M_{\mathrm{cp}}\right) \times 1}$. For any $k, p \in[1, K]$, it follows $\mathbf{b}_{k}^{H} \mathbf{b}_{k}=1$ and $\mathbf{b}_{k}^{H} \mathbf{b}_{p}=0, \forall p \neq k$. The time-domain CIR samples of the $l^{\prime}$-th path are rearranged into the vector

$$
\mathbf{h}_{l^{\prime}}=\left[h\left[0, l^{\prime}\right], h\left[1, l^{\prime}\right], \cdots, h\left[M N+M_{\mathrm{cp}}-1, l^{\prime}\right]\right]^{T},
$$

which is projected into $\mathrm{V} 1$ as

$$
\mathbf{h}_{l^{\prime}}=\sum_{k=1}^{K} c_{l^{\prime}, k} \mathbf{b}_{k}+\mathbf{e}_{\mathrm{T}}^{l^{\prime}}
$$

where $\mathbf{e}_{\mathrm{T}}^{l^{\prime}}$ is the modeling error corresponding to the CIR of $l^{\prime}$-th path and takes the same form as $\mathbf{h}_{l^{\prime}}$. For brevity, a time-index set is defined as $\mathrm{Z}=\left\{M_{\mathrm{cp}}, \cdots, M+M_{\mathrm{cp}}-\right.$ $\left.1, \cdots,(N-1) M+M_{\mathrm{cp}}, \cdots, N M+M_{\mathrm{cp}}-1\right\}$. Relying on (8), we have

$$
\mathbf{h}_{l^{\prime}}^{\mathrm{Z}}=\sum_{k=1}^{K} c_{l^{\prime}, k} \mathbf{b}_{k}^{\mathrm{Z}}+\mathbf{e}_{\mathrm{T}}^{l^{\prime}, \mathrm{Z}}
$$

where $\mathbf{h}_{l^{\prime}}^{\mathrm{Z}}=\left[h\left[M_{\mathrm{cp}}, l^{\prime}\right], \cdots, h\left[M N+M_{\mathrm{cp}}-1, l^{\prime}\right]\right]^{T} \in$ $\mathbb{C}^{M N \times 1} ; \mathbf{b}_{k}^{\mathrm{Z}}$ and $\mathbf{e}_{T}^{l^{\prime}, \mathrm{Z}}$ take the same form as $\mathbf{h}_{l^{\prime}}^{\mathrm{Z}}$.

For the $l^{\prime}$-th path, the ECRs within one OTFS transmission period are rewritten as

$$
\boldsymbol{\Omega}_{l^{\prime}}=\left[\boldsymbol{\Omega}_{l^{\prime}}^{0}, \cdots, \boldsymbol{\Omega}_{l^{\prime}}^{v^{\prime}}, \cdots, \boldsymbol{\Omega}_{l^{\prime}}^{N-1}\right]^{T} \in \mathbb{C}^{M N \times 1},
$$

where $\Omega_{l^{\prime}}^{v^{\prime}}=\left[\Omega\left\{0, l^{\prime}, v^{\prime}\right\}, \cdots, \Omega\left\{M-1, l^{\prime}, v^{\prime}\right\}\right]^{T} \in$ $\mathbb{C}^{M \times 1}$. By using 2 and 9 ,, $\boldsymbol{\Omega}_{l^{\prime}}$ is derived as

$\boldsymbol{\Omega}_{l^{\prime}}=\frac{1}{\sqrt{N}} \sum_{k=1}^{K} c_{l^{\prime}, k}\left(\mathbf{F}_{N} \otimes \mathbf{I}_{M}\right) \mathbf{b}_{k}^{\mathrm{Z}}+\frac{1}{\sqrt{N}}\left(\mathbf{F}_{N} \otimes \mathbf{I}_{M}\right) \mathbf{e}_{\mathrm{T}}^{l^{\prime}, \mathrm{Z}}$.

Proposition 2: Defining the $k$-th transform-domain basis function as

$$
\mathbf{a}_{k} \triangleq \frac{1}{\sqrt{N}}\left(\mathbf{F}_{N} \otimes \mathbf{I}_{M}\right) \mathbf{b}_{k}^{\mathrm{Z}} \in \mathbb{C}^{M N \times 1},
$$

the ECRs of the $l^{\prime}$-th path is modeled by

$$
\boldsymbol{\Omega}_{l^{\prime}}=\sum_{k=1}^{K} c_{l^{\prime}, k} \mathbf{a}_{k}+\mathbf{e}_{\mathrm{D}}^{l^{\prime}}
$$

in the subspace

$$
\mathrm{V} 2: \operatorname{span}\left\{\mathbf{a}_{1}, \mathbf{a}_{2}, \cdots, \mathbf{a}_{K}\right\},
$$

where $\mathbf{e}_{\mathrm{D}}^{l^{\prime}}$ is the modeling error of the $l^{\prime}$-th path, i.e.,

$$
\mathbf{e}_{\mathrm{D}}^{l^{\prime}} \triangleq \frac{1}{\sqrt{N}}\left(\mathbf{F}_{N} \otimes \mathbf{I}_{M}\right) \mathbf{e}_{\mathrm{T}}^{l^{\prime}, \mathrm{Z}} \in \mathbb{C}^{M N \times 1}
$$

At the end, the relation between $(6$ and $[13$ can be realized by defining

$\mathbf{a}_{k} \triangleq\left[A(0, k, 0), \cdots, A\left(l, k, v^{\prime}\right), \cdots, A(M-1, k, N-1)\right]^{T}$,

where

$$
A\left(l, k, v^{\prime}\right)=\frac{1}{N} \sum_{i=0}^{N-1} b\left[i M+M_{\mathrm{cp}}+l, k\right] e^{\frac{-j 2 \pi i v^{\prime}}{N}} .
$$


Relying on (13), the OTFS channel is modeled in the lowdimensional subspace V2. For the whole $L$ channel paths, the number of unknowns is $K \times L(K \ll M)$, which is obviously less than the number of the ERCs, i.e., $M \times L \times N$.

\section{B. Analysis of Channel Modeling Accuracy}

We analyze the accuracy of subspace channel modeling by evaluating the square bias of the modeling error, which provides us some insights into the selection of subspace dimension $K$. Specifically, the square bias of the modeling error is defined as

$$
\xi_{\mathrm{D}} \triangleq \frac{1}{L} \sum_{l^{\prime}=0}^{L-1} \xi_{\mathrm{D}}^{l^{\prime}},
$$

where $\xi_{\mathrm{D}}^{l^{\prime}}$ is the the square bias of $\mathbf{e}_{\mathrm{D}}^{l^{\prime}}$, i.e.,

$$
\xi_{\mathrm{D}}^{l^{\prime}} \triangleq \frac{1}{M N} \mathbb{E}\left\{\left(\mathbf{e}_{\mathrm{D}}^{l^{\prime}}\right)^{H} \mathbf{e}_{\mathrm{D}}^{l^{\prime}}\right\} .
$$

Substituting (15) into (19), we have

$$
\xi_{\mathrm{D}}^{l^{\prime}} \triangleq \frac{1}{N} \xi_{\mathrm{T}}^{l^{\prime}}
$$

where $\xi_{\mathrm{T}}^{l^{\prime}} \triangleq \frac{1}{M N} \mathbb{E}\left\{\left(\mathbf{e}_{\mathrm{T}}^{l^{\prime}, Z}\right)^{H} \mathbf{e}_{\mathrm{T}}^{l^{\prime}, Z}\right\}$ is defined as the square bias of $\mathbf{e}_{\mathrm{T}}^{l^{\prime}, \mathrm{Z}}$, which can be rewritten as $\xi_{\mathrm{T}}^{l^{\prime}}=$ $\frac{1}{M N+M_{\mathrm{cp}}} \mathbb{E}\left\{\left(\mathbf{e}_{\mathrm{T}}^{l^{\prime}}\right)^{H} \mathbf{e}_{\mathrm{T}}^{l^{\prime}}\right\}$. From 20 , the square bias of the modeling error of $\boldsymbol{\Omega}_{l^{\prime}}$ depends on $\xi_{\mathrm{T}}^{l}$. The value of $\xi_{\mathrm{T}}^{l^{\prime}}$ directly relies on the selection of $\mathbf{b}_{k}$ and $K$. In this paper, the Slepian sequences are adopted as time-domain basis functions thanks to their superior channel fitting performance $[\overline{8}]$. Specifically, the sequences are the eigenvectors of matrix $\boldsymbol{\Theta}$

$$
\Theta \mathbf{b}_{k}=\lambda_{k} \mathbf{b}_{k},
$$

where the entries of $\Theta$ are

$$
\Theta(a, b)=\frac{\sin \left[2 \pi(a-b) f_{d} T_{s}\right]}{\pi(a-b)},
$$

$a, b \in\left\{0,1, \cdots, M N+M_{\mathrm{cp}}-1\right\}$.

When the number of dimension $K=M_{\mathrm{total}} \triangleq M N+M_{\mathrm{cp}}$, V1 becomes a completely orthogonal space. For arbitrary channel vector $\mathbf{h}_{l^{\prime}}$, it can be represented by

$$
\mathbf{h}_{l^{\prime}}=\sum_{k=1}^{M_{\text {total }}} c_{l^{\prime}, k} \mathbf{b}_{k},
$$

where $c_{l^{\prime}, k}=\mathbf{b}_{k}^{H} \mathbf{h}_{l^{\prime}}$. Combing [8] and 23,, $\mathbf{e}_{\mathrm{T}}^{l^{\prime}}$ is rewritten as

$$
\mathbf{e}_{\mathrm{T}}^{l^{\prime}}=\mathbf{h}_{l^{\prime}}-\sum_{k=1}^{K} c_{l^{\prime}, k} \mathbf{b}_{k}=\sum_{k=K+1}^{M_{\text {total }}} c_{l^{\prime}, k} \mathbf{b}_{k} .
$$

Substituting 24, into 20, $\xi_{\mathrm{D}}^{l^{\prime}}$ is expressed as

$$
\begin{aligned}
\xi_{\mathrm{D}}^{l^{\prime}} & =\frac{1}{N M_{\text {total }}} \mathbb{E}\left\{\sum_{k=K+1}^{M_{\text {total }}} \mathbf{b}_{k}^{H} \mathbf{h}_{l^{\prime}} \mathbf{h}_{l^{\prime}}^{H} \mathbf{b}_{k}\right\} \\
& =\frac{1}{N M_{\text {total }}} \sum_{k=K+1}^{M_{\text {total }}} \mathbf{b}_{k}^{H} \mathbf{R}_{h}^{l^{\prime}} \mathbf{b}_{k} .
\end{aligned}
$$

where $\mathbf{R}_{h}^{l^{\prime}}$ is the covariance matrix of the $l^{\prime}$-th channel path. According to (25), the theoretical modeling error of the $l^{\prime}$-th path depends on: OTFS transmission block size $M N$, basis functions $\left\{\mathbf{b}_{k}\right\}$, channel correlation matrix of the $l^{\prime}$-th path $\mathbf{R}_{h}^{l^{\prime}}$, and subspace dimension $K$. More importantly, in practical wireless transmission scenarios, the channel correlation function $r(\tau)$ can be diverse, which means $\mathbf{R}_{h}^{l^{\prime}}$ does not have an inherent attribute. Hence, the subspace dimension $K$, corresponding to the modeling error, should be properly designed targeting the specific propagation environments.

The Clarke channel model, which has a continuous " $U$ shaped" Doppler spectrum, has been verified by the existing measurement campaigns [29], [33], [34], yielding its application into extensive wireless communication scenarios. In Sec. III-C, we will select the classic Clarke channel to analyze the accuracy of subspace channel modeling, which can show the advantages of the proposed scheme. Note that for the other cases of CDSC, our measurements can be also used.

\section{Modeling Error in Clarke Channel}

In classical Clarke channel, $h\left[n, l^{\prime}\right]$ follows Rayleigh distribution in time, of which the autocorrelation function is

$\mathbb{E}\left\{h\left[n, l^{\prime}\right] h^{*}\left[m, l^{\prime}\right]\right\}=\sigma_{l^{\prime}}^{2} \delta\left(l^{\prime}-l^{\prime \prime}\right) J_{0}\left(2 \pi f_{d}|m-n| T_{s}\right)$.

Hence, for the $(u, t)$-th element in $\mathbf{R}_{h}^{l^{\prime}}$, we have

$$
R_{h}^{l^{\prime}}(u, t)=\sigma_{l^{\prime}}^{2} J_{0}\left(2 \pi f_{d}|u-t| T_{s}\right), u, t \in\left[0, M_{\text {total }}-1\right] .
$$

Using (25) and (26), the square bias of the modeling error $\xi_{\mathrm{D}}$ in $(18)$ is derived as

$$
\begin{aligned}
\xi_{\mathrm{D}} & =\frac{1}{L N M_{\text {total }}} \sum_{l^{\prime}=0}^{L-1} \sigma_{l^{\prime}}^{2} \sum_{k=K+1}^{M_{\text {total }}} \mathbf{b}_{k}^{H} \mathbf{R}_{h} \mathbf{b}_{k} \\
& =\frac{1}{L N M_{\text {total }}} \sum_{k=K+1}^{M_{\text {total }}} \mathbf{b}_{k}^{H} \mathbf{R}_{h} \mathbf{b}_{k},
\end{aligned}
$$

where $\mathbf{R}_{h}$ is composed of $R_{h}(u, t)=J_{0}\left(2 \pi f_{d}|u-t| T_{s}\right)$, $u, t \in\left[0, M_{\text {total }}-1\right]$.

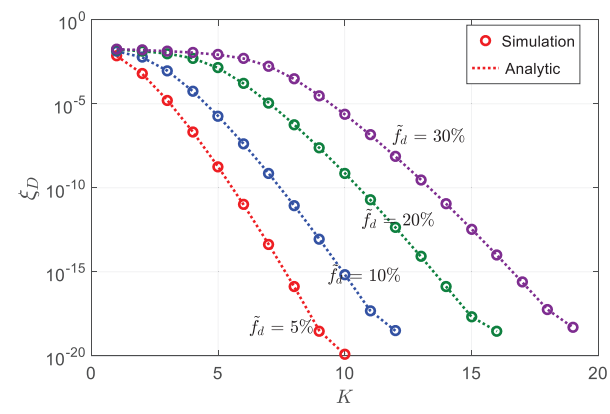

Fig. 6. The square bias $\xi_{D}$ comparison of dimension $K$. The velocities are $137,270,550$ and $820 \mathrm{~km} / \mathrm{h}$, corresponding to $\tilde{f}_{d}=5 \%, 10 \%, 20 \%$ and $30 \%$, respectively.

Fig. 6 compares the square bias $\xi_{\mathrm{D}}$ versus the subspace dimension $K$. As $K$ increases, $\xi_{\mathrm{D}}$ decreases quickly. Given $\xi_{\mathrm{D}}$ less than $10^{-10}$, the least required orders for velocity $=137,270,550$ and $820 \mathrm{~km} / \mathrm{h}$ cases are $6,8,11$ and 14 , 


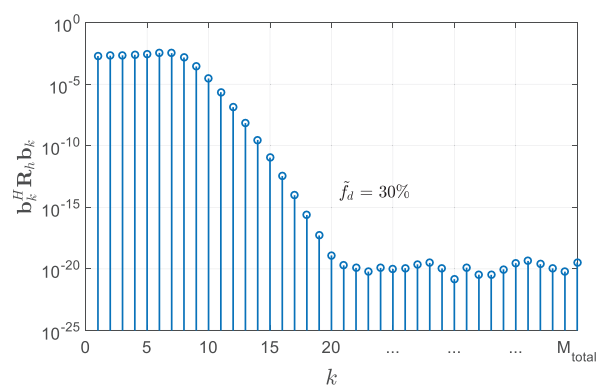

Fig. 7. The distribution of $\mathbf{b}_{k}^{H} \mathbf{R}_{h} \mathbf{b}_{k}$ in 27, when $\tilde{f}_{d}=30 \%$.

respectively. Additionally, by (27), we plot the distribution of $\mathbf{b}_{k}^{H} \mathbf{R}_{h} \mathbf{b}_{k}$ in Fig. 7, which can be treated as the modeling error at the $k$-th dimension of the subspace. As we can see, in high dimensions, e.g., $k>20$, the value of $\mathbf{b}_{k}^{H} \mathbf{R}_{h} \mathbf{b}_{k}$ is so small that can be neglected.

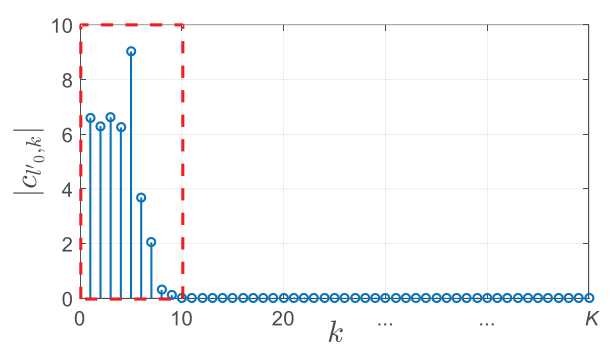

(a) The distribution of the projection coefficients of the $l_{0}^{\prime}$-th path: $c_{l_{0}^{\prime}, k}$.



(b) The subspace DD channel modeling at the $l_{0}^{\prime}$-th delay and $v_{0}^{\prime}$-th Doppler indices $\Omega\left\{l, l_{0}^{\prime}, v_{0}^{\prime}\right\}$.

Fig. 8. The distribution of the projection coefficients and the modeling results of the ECRs in the subspace V2, where $\tilde{f}_{d}=20 \%$, corresponding to a velocity of $550 \mathrm{~km} / \mathrm{h}$.

Fig. 8 gives an example to show the modeling results of the OTFS channel (path $l_{0}^{\prime}$ ) when $\tilde{f}_{d}=20 \%$. The distribution of the projection coefficients $c_{l_{0}^{\prime}, k}$ in $\mathrm{V} 2$ is demonstrated in Fig. 8(a), where the components for $k>10$ can be neglected. Substituting the first ten coefficients $c_{l_{0}^{\prime}, k}, k=1,2, \cdots, 10$ into (6), the ECRs $\Omega\left\{l, l_{0}^{\prime}, v_{0}^{\prime}\right\}, l \in[0, M-1]$ can be perfectly reconstructed in Fig. 8(b) ${ }^{4}$ As we can see, by leveraging the subspace channel modeling, the original channel responses of the $l_{0}^{\prime}$-th path $\Omega_{l_{0}^{\prime}}$ can be approximated by only utilizing a small number of the projection coefficients. In other words,

\footnotetext{
${ }^{4}$ Here, limited by space, we plot the fitting curve of the channel response at the $l_{0}^{\prime}$-th delay and $v_{0}^{\prime}$-th Doppler indices. Actually, utilizing these ten coefficients $c_{l_{0}^{\prime}, k}, k=1,2, \cdots, 10$, the overall responses of the $l_{0}^{\prime}$-th path, i.e., $\boldsymbol{\Omega}_{l_{0}^{\prime}}$, can be ideally reconstructed by 13 .
}

the dimension of the unknown gains in $\Omega\left(l, l_{0}^{\prime}, v^{\prime}\right)$ can be reduced from $M N$ to $K$.

Benefiting from the precisely subspace channel modeling, the DD CE can be implemented by estimating the projection coefficients in V2. For all transmission paths, the number of the projection coefficients that need to be estimated is $K L$, which is much less than the size of OTFS transmission block $M N$. Thus, by inserting the pilots into the OTFS transmission block, where the number of pilots is not less than $K L$, the projection coefficients can be acquired by solving an overdetermined least squares (LS) problem in Section IV.

\section{Channel Estimation in the Subspace}

In order to estimate the projection coefficients of ECRs in V2, the training pilots are inserted into the OTFS transmission frame. As shown in Fig. 9, a CIPP is proposed, which aims to achieve the relatively low pilot overhead and satisfactory $\mathrm{CE}$ performance. At each corner of transmission frame, the pilots occupy $M_{\mathrm{P}} \times N_{\mathrm{P}}$ grids, where $M_{\mathrm{P}} \ll M$ and $N_{\mathrm{P}} \ll N$. In the Doppler direction, to avoid the interference between the pilots and data, the guard interval (GI) with length $N_{\mathrm{GI}}$ is inserted at the both sides of pilot block. The value of $N_{\mathrm{GI}}$ depends on the channel maximum Doppler frequency $f_{d}$, which follows $N_{\mathrm{GI}}>2\left\lfloor N f_{d} / \Delta f\right\rfloor$. Then, the rest grids with number of $N_{\text {data }}=N-2 N_{\mathrm{P}}-2 N_{\mathrm{GI}}$ in the Doppler direction are used for assigning data symbols. In the delay direction, to protect the data symbols from interference generated by the pilots, the GI with length $M_{\mathrm{GI}}\left(M_{\mathrm{GI}} \geq L-1\right)$ is inserted at the both sides of data block. Note that the values of $N_{\mathrm{GI}}$, $N_{\mathrm{P}}$ and $N_{\text {data }}$ can be changeable when considering different OTFS transmission block size and channel Doppler spread. It would result in different shapes of CIPP, which will be detailed in Sec. VI.



Fig. 9. The developed CIPP in OTFS system.

From Fig. 9, the resource grids for mapping pilot symbols and data symbols are

$$
\text { Pis }=4 M_{\mathrm{P}} N_{\mathrm{P}},
$$

and

$$
\text { Das }=M_{\text {data }} N+2 N_{\text {data }}\left(M_{\mathrm{P}}+M_{\mathrm{GI}}\right),
$$

respectively. We define the transmission efficiency for OTFS system as

$$
\eta=\frac{D a s}{M N} \times 100 \% .
$$


Before projection coefficient estimation, we formulate the OTFS signal transmission in the proposed subspace.

Proposition 3: The received DD-domain vector $\mathbf{y}=$ $\operatorname{vec}\left(\mathbf{Y}^{T}\right) \in \mathbb{C}^{M N \times 1}$ is expressed as

$$
\mathbf{y}=\mathbf{S c}+\mathbf{w},
$$

where $\mathbf{w}$ is the noise vector and takes the same form of $\mathbf{y}$, and $\mathbf{c}$ denotes the projection coefficients, i.e.,

$$
\begin{aligned}
& \mathbf{c}=\left[\mathbf{c}_{0}^{T}, \cdots, \mathbf{c}_{l^{\prime}}^{T}, \cdots, \mathbf{c}_{L-1}^{T}\right]^{T} \in \mathbb{C}^{K L \times 1}, \\
& \mathbf{c}_{l^{\prime}}=\left[c_{l^{\prime}, 1}, \cdots, c_{l^{\prime}, k}, \cdots, c_{l^{\prime}, K}\right]^{T} \in \mathbb{C}^{K \times 1} .
\end{aligned}
$$

In (31), $\mathbf{S}$ consists of the transmitted DD-domain data that coupled with the subspace basis functions:

$$
\mathbf{S}=\left[\begin{array}{c}
\overline{\mathbf{X}}_{0}\left(\mathbf{I}_{L} \otimes \mathbf{A}_{0}\right) \\
\vdots \\
\overline{\mathbf{X}}_{M-1}\left(\mathbf{I}_{L} \otimes \mathbf{A}_{M-1}\right)
\end{array}\right] \in \mathbb{C}^{M N \times K L},
$$

where

$$
\mathbf{A}_{l}=\left[\begin{array}{ccc}
A(l, 1,0) & \cdots & A(l, K, 0) \\
\vdots & \ddots & \vdots \\
A(l, 1, N-1) & \cdots & A(l, K, N-1)
\end{array}\right] \in \mathbb{C}^{N \times K} .
$$

In $\sqrt{33},, \overline{\mathbf{X}}_{l}, l \in[0, M-1]$ follows

$$
\overline{\mathbf{X}}_{l}=\left[\left(\overline{\mathbf{X}}_{l}(0,:)\right)^{T}, \cdots,\left(\overline{\mathbf{X}}_{l}(N-1,:)\right)^{T}\right]^{T} \in \mathbb{C}^{N \times N L},
$$

where $\overline{\mathbf{X}}_{l}(n,:)=\left[\operatorname{Circ}\left(\overline{\mathbf{x}}_{l, 0}, n-1\right), \cdots, \operatorname{Circ}\left(\overline{\mathbf{x}}_{l, L-1}, n-1\right)\right] \in$ $\mathbb{C}^{1 \times N L}$ and $\overline{\mathbf{x}}_{l, l^{\prime}}=\left[X\left(\left(l-l^{\prime}\right)_{M}, 0\right), \cdots, X\left(\left(l-l^{\prime}\right)_{M}, 1\right)\right] \in$ $\mathbb{C}^{1 \times N}$

\section{A. Projection Coefficient Estimation}

The purpose of coefficient estimation is to obtain $\mathbf{c}$ in (31), relying on the training pilots. According to the CIPP in Fig. 9 and Proposition 3, we can formulate the transmission that only corresponding to pilot symbols. To help analyze, we define two matrices, i.e., $\mathbf{F}_{\mathrm{P}}^{\mathrm{Tx}} \in \mathbb{C}^{M \times N}$ and $\mathbf{F}_{\mathrm{P}}^{\mathrm{Rx}} \in \mathbb{C}^{M \times N}$ illustrated in Fig. 10 Matrix $\mathbf{F}_{\mathrm{P}}^{\mathrm{Tx}}$ is to pick out the pilots at the transmitter side, i.e,

$$
\mathbf{X}^{\text {pilot }}=\mathbf{F}_{\mathrm{P}}^{\mathrm{Tx}} \odot \mathbf{X} .
$$

At the receiver side, due to the channel delay and Doppler spreads, the transmitted pilot symbols will dispersive along both delay and Doppler directions. Accordingly, we expand the range of "nonzero area" in $\mathbf{F}_{\mathrm{P}}^{\mathrm{Tx}}$. The increased lengths in delay and Doppler directions are $M_{\mathrm{D}}$ and $N_{\mathrm{B}}$, respectively. For the received pilot symbols, we have

$$
\mathbf{Y}^{\text {pilot }}=\mathbf{F}_{\mathrm{P}}^{\mathrm{Rx}} \odot \mathbf{Y} .
$$

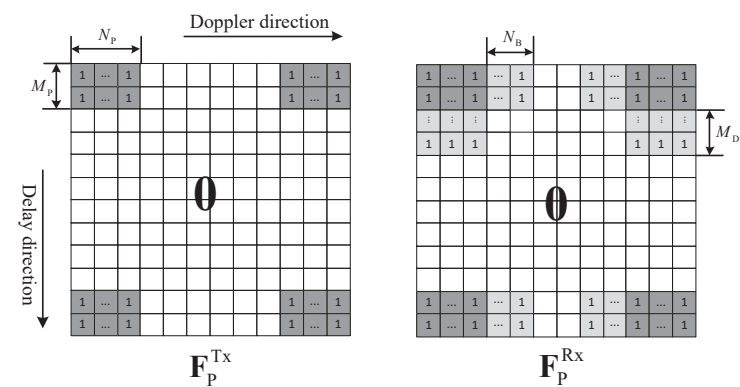

Fig. 10. The illustration of matrices $\mathbf{F}_{\mathrm{P}}^{\mathrm{Tx}}$ and $\mathbf{F}_{\mathrm{P}}^{\mathrm{Rx}}$.

Note that the values of $M_{\mathrm{D}}$ and $N_{\mathrm{B}}$ should be properly considered. On the one hand, the bigger values of $M_{\mathrm{D}}$ and $N_{\mathrm{B}}$ are, the more observation symbols we can obtain. As a result, it helps to improve the CE accuracy. In contrast, with relatively large values of $M_{\mathrm{D}}$ and $N_{\mathrm{B}}$, the more interference components will be involved by data symbols, deteriorating the $\mathrm{CE}$ accuracy. Hence, to tradeoff the observations and interference, we consider

$$
\begin{aligned}
& 0 \leq M_{\mathrm{D}} \leq L-1, \\
& 0 \leq N_{B} \leq \min \left\{\left\lfloor N f_{d} / \Delta f\right\rfloor, N_{\mathrm{GI}}-\left\lceil N f_{d} / \Delta f\right\rceil\right\} .
\end{aligned}
$$

Relying on (31), we have

$$
\mathbf{y}^{\mathrm{P}}=\mathbf{S}^{\mathrm{P}} \mathbf{c}+\chi^{\mathrm{P}}+\mathbf{w}^{\mathrm{P}},
$$

where

$$
\mathbf{y}^{\mathrm{P}}=\operatorname{vec}\left(\left(\mathbf{X}^{\mathrm{pilot}}\right)^{T}\right)=\operatorname{vec}\left(\left(\mathbf{F}_{\mathrm{P}}^{\mathrm{Rx}} \odot \mathbf{Y}\right)^{T}\right),
$$

$\chi^{\mathrm{P}}$ and $\mathrm{w}^{\mathrm{P}}$ denote the interference and noise vector, respectively. They take the same form as $\mathbf{y}^{\mathrm{P}}$. In $\sqrt[40]{ }, \mathrm{S}^{\mathrm{P}}$ is defined as

$$
\mathbf{S}^{\mathrm{P}}=\left[\begin{array}{c}
\overline{\mathbf{X}}_{0}^{\mathrm{P}}\left(\mathbf{I}_{L} \otimes \mathbf{A}_{0}\right) \\
\vdots \\
\overline{\mathbf{X}}_{M-1}^{\mathrm{P}}\left(\mathbf{I}_{L} \otimes \mathbf{A}_{M-1}\right)
\end{array}\right]
$$

where $\overline{\mathbf{X}}_{l}^{\mathrm{P}}, l \in[0, M-1]$ can be found from (43)-(45).

We do not propose to estimate the coefficients $\mathbf{c}$ from 440 , since the dimension of $y^{\mathrm{P}}$ is quite large. Fortunately, the number of nonzero elements in $\mathbf{y}^{\mathrm{P}}$ is $Q=4\left(N_{\mathrm{P}}+N_{\mathrm{B}}\right) M_{\mathrm{P}}+$ $2 M_{\mathrm{D}} N_{\mathrm{P}}$, which follows $Q \ll M N$. Hence, we use a $Q \times M N$ matrix $\mathbf{T}$ to multiply $\mathbf{y}^{\mathrm{P}}$, picking out the nonzero entries. By doing so, the dimension of $\mathrm{y}^{\mathrm{P}}$ can be significantly decreased. Recalling (40), we have

$$
\mathbf{T} \mathbf{y}^{\mathrm{P}}=\mathbf{T} \mathbf{S}^{\mathrm{P}} \mathbf{c}+\mathbf{T} \chi^{\mathrm{P}}+\mathbf{T} \mathbf{w}^{\mathrm{P}} .
$$

Defining $\tilde{\mathbf{y}}^{\mathrm{P}}=\mathbf{T} \mathbf{y}^{\mathrm{P}} \in \mathbb{C}^{Q \times 1}, \tilde{\mathbf{S}}^{\mathrm{P}}=\mathbf{T S}^{\mathrm{P}} \in \mathbb{C}^{Q \times K L}, \tilde{\chi}^{\mathrm{P}}=$ $\mathbf{T} \chi^{\mathrm{P}} \in \mathbb{C}^{Q \times 1}$ and $\tilde{\mathbf{w}}^{\mathrm{P}}=\mathbf{T} \mathbf{w}^{\mathrm{P}} \in \mathbb{C}^{Q \times 1}, 46$ is rewritten as

$$
\tilde{\mathbf{y}}^{\mathrm{P}}=\tilde{\mathbf{S}}^{\mathrm{P}} \mathbf{c}+\tilde{\boldsymbol{\chi}}^{\mathrm{P}}+\tilde{\mathbf{w}}^{\mathrm{P}} .
$$

$$
\begin{gathered}
\overline{\mathbf{X}}_{l}^{\mathrm{P}}=\left\{\left(\overline{\mathbf{X}}_{l}^{\mathrm{P}}(0,:)\right)^{T}, \cdots,\left(\overline{\mathbf{X}}_{l}^{\mathrm{P}}(n,:)\right)^{T}, \cdots,\left(\overline{\mathbf{X}}_{l}^{\mathrm{P}}(N-1,:)\right)^{T}\right\}^{T} \in \mathbb{C}^{N \times N L} \\
\overline{\mathbf{X}}_{l}^{\mathrm{P}}(n,:)=\left[\operatorname{Circ}\left(\overline{\mathbf{x}}_{l, 0}^{\mathrm{P}}, n-1\right), \cdots, \operatorname{Circ}\left(\overline{\mathbf{x}}_{l, l^{\prime}}^{\mathrm{P}}, n-1\right), \cdots, \operatorname{Circ}\left(\overline{\mathbf{x}}_{l, L-1}^{\mathrm{P}}, n-1\right)\right] \in \mathbb{C}^{1 \times N L} \\
\overline{\mathbf{x}}_{l, l^{\prime}}^{\mathrm{P}}=\mathbf{F}_{\mathrm{P}}^{\mathrm{Tx}}\left(\left(l-l^{\prime}\right)_{M},:\right) \odot\left[X\left(\left(l-l^{\prime}\right)_{M}, 0\right), X\left(\left(l-l^{\prime}\right)_{M}, N-1\right), \cdots, X\left(\left(l-l^{\prime}\right)_{M}, 1\right)\right] \in \mathbb{C}^{1 \times N}
\end{gathered}
$$


The LMMSE method is performed to estimate $\mathbf{c}$

$$
\hat{\mathbf{c}}_{\mathrm{mmse}}=\boldsymbol{\Lambda}_{\mathrm{mmse}} \tilde{\mathbf{y}}^{\mathrm{P}},
$$

where

$$
\boldsymbol{\Lambda}_{\text {mmse }}=\left(\left(\tilde{\mathbf{S}}^{\mathrm{P}}\right)^{H} \tilde{\mathbf{S}}^{\mathrm{P}}+\sigma^{2}\left(\mathbf{R}_{\mathbf{c}}\right)^{-1}\right)^{-1}\left(\tilde{\mathbf{S}}^{\mathrm{P}}\right)^{H} .
$$

In (49), $\mathbf{R}_{\mathbf{c}}$ is the covariance matrix of $\mathbf{c}$, which follows

$$
\mathbf{R}_{\mathbf{c}}=\operatorname{blkdiag}\left\{\mathbf{R}_{\mathbf{c}_{0}}, \cdots, \mathbf{R}_{\mathbf{c}_{l^{\prime}}}, \cdots, \mathbf{R}_{\mathbf{c}_{L-1}}\right\} \in \mathbb{C}^{K L \times K L} .
$$

where $\mathbf{R}_{\mathbf{c}_{l^{\prime}}}=\mathbf{B}^{H} \mathbf{R}_{\Omega_{l^{\prime}}} \mathbf{B}$ and $\mathbf{B}=\left[\mathbf{a}_{1}, \cdots, \mathbf{a}_{K}\right]$.

\section{B. Channel Estimation Accuracy}

We evaluate the $\mathrm{CE}$ accuracy by investigating the mean squared error (MSE). Based on the estimated projection coefficients $\hat{\mathbf{c}}$, the whole ECRs $\boldsymbol{\Omega}=$ $\left[\boldsymbol{\Omega}_{0}^{H}, \cdots, \boldsymbol{\Omega}_{l^{\prime}}^{H}, \cdots, \boldsymbol{\Omega}_{L-1}^{H}\right] \in \mathbb{C}^{M N L \times 1}$ are reconstructed by

$$
\hat{\mathbf{\Omega}}=\left(\mathbf{I}_{L} \otimes \mathbf{B}\right) \hat{\mathbf{c}} .
$$

The MSE of CE is defined as

$$
\mathrm{MSE} \triangleq \frac{1}{M N L} \mathbb{E}\left\{\|\boldsymbol{\Omega}-\hat{\boldsymbol{\Omega}}\|^{2}\right\} .
$$

With (13) and 52), MSE is calculated by

$$
\begin{aligned}
\mathrm{MSE} & =\frac{1}{M N L} \mathbb{E}\left\{\left\|\left(\mathbf{I}_{L} \otimes \mathbf{B}\right)(\hat{\mathbf{c}}-\mathbf{c})+\mathbf{e}_{\mathrm{D}}\right\|^{2}\right\} \\
& =\frac{1}{M N L} \mathbb{E}\left\{\left\|\mathbf{e}_{\text {est }}+\mathbf{e}_{\mathrm{D}}\right\|^{2}\right\},
\end{aligned}
$$

where $\mathbf{e}_{\mathrm{D}}=\left[\left(\mathbf{e}_{\mathrm{D}}^{0}\right)^{T}, \cdots,\left(\mathbf{e}_{\mathrm{D}}^{L-1}\right)^{T}\right]^{T} \in \mathbb{C}^{M N L \times 1}$ and

$$
\mathbf{e}_{\text {est }}=\left(\mathbf{I}_{L} \otimes \mathbf{B}\right)(\hat{\mathbf{c}}-\mathbf{c}) \in \mathbb{C}^{M N L \times 1} .
$$

Note that the CE error consists of two parts: 1) The modeling error for all paths $\left(\mathbf{e}_{\mathrm{D}}\right)$ in the constructed subspace and $2)$ The estimation error $\left(\mathbf{e}_{\text {est }}\right)$ introduced by the estimated coefficients. They are uncorrelated to each other. According to the analysis of the channel modeling error in Section III-B, (53) can be rewritten as

$$
\mathrm{MSE}=\psi_{\mathrm{D}}+\xi_{\mathrm{D}}
$$

where $\xi_{\mathrm{D}}$ is the square bias of modeling error, shown in (27), and $\psi_{\mathrm{D}}$ denotes the variance of estimation error, i.e.,

$$
\psi_{\mathrm{D}}=\frac{1}{M N L} \mathbb{E}\left\{\left\|\mathbf{e}_{\mathrm{est}}\right\|^{2}\right\}
$$

In the following part, we focus on analyzing $\psi_{\mathrm{D}}$.

Recalling (47), the received pilot signal suffers from both noise and interference. Due to the channel Doppler spread, the data symbols diffuse along the Doppler dimension, thus involving the interference $\tilde{\chi}^{\mathrm{P}}$. It is difficult to theoretically evaluate the influence of $\tilde{\chi}^{\mathrm{P}}$ to the CE accuracy. Hence, we propose to derive a lower bound, which is obtained by considering $\tilde{\chi}^{\mathrm{P}}=\mathbf{0}$. Or rather, the lower bound corresponds to the case that $N_{\text {data }}$ in Fig. 9 is zero. Thus, the variance of the estimation error $\psi_{\mathrm{D}}$ is bounded by $\psi_{\mathrm{D}}^{\prime}$, i.e.,

$$
\psi_{\mathrm{D}} \geq \psi_{\mathrm{D}}^{\prime},
$$

where $\psi_{\mathrm{D}}^{\prime}$ is derived as

$$
\begin{gathered}
\psi_{D}^{\prime}=\frac{1}{M N L} \operatorname{Tr}\left\{\left(\boldsymbol{\Lambda}_{\mathrm{mmse}} \tilde{\mathbf{S}}^{\mathrm{P}}-\mathbf{I}_{K L}\right) \mathbf{R}_{\mathbf{c}}\left(\boldsymbol{\Lambda}_{\mathrm{mmse}} \tilde{\mathbf{S}}^{\mathrm{P}}-\mathbf{I}_{K L}\right)^{H}\right. \\
\left.+\frac{1}{\sigma^{2}} \boldsymbol{\Lambda}_{\mathrm{mmse}} \boldsymbol{\Lambda}_{\mathrm{mmse}}^{H}\right\} .
\end{gathered}
$$

Hence, according to 56 and 58, the MSE of CE is bounded by

$$
\mathrm{MSE} \geq \psi_{\mathrm{D}}^{\prime}+\xi_{\mathrm{D}} .
$$

As analyzed above, the CE accuracy depends on the design of subspace channel modeling and estimation of projection coefficients. In the subspace channel modeling, the dimension $K$ should be set as a appropriate value, not as big as possible. Although the bigger value of $K$ can achieve a more accurate modeling result, it also involves more unknown projection coefficients. According to $(59)$ and $(60)$, estimating the projection coefficients with a large amount deteriorates the $\mathrm{CE}$ accuracy. On the other hand, for the projection coefficient estimation, we still have to face a challenge that how to arrange the pilot symbols with limited overhead, thus achieving a satisfactory CE accuracy. The above issues will be addressed in Sec. VI.

\section{Computational Complexity of Coefficient Estimation}

The computational complexity of the coefficient estimation is analyzed in terms of the number of complex multiplications (CMs). The main calculational overhead of acquiring the coefficients $\hat{\mathbf{c}}_{\text {mmse }}$, derives from the calculation of $\boldsymbol{\Lambda}_{\text {mmse }}$ in (49). If the pilot sequences in each OTFS transmission period are changeable, it will cause calculational burden due to the update of $\tilde{\mathbf{S}}^{\mathrm{P}}$ in 49 . However, in practice, we can set the pilot sequences are fixed, which means $\tilde{\mathbf{S}}^{\mathrm{P}}$ is constant rather variable. Note that using the fixed pilot sequences will not affect the proposed CE scheme. Hence, in this way, the computational complexity of obtaining $\hat{\mathbf{c}}_{\text {mmse }}$ can be significantly decreased. Specifically, obtaining $\hat{\mathbf{c}}_{\mathrm{mmse}}$ in 48 requires $Q K L=\left(4\left(N_{\mathrm{P}}+N_{\mathrm{B}}\right) M_{\mathrm{P}}+2 M_{\mathrm{D}} N_{\mathrm{P}}\right) K L \mathrm{CMs}$. The dimension of subspace: $K$ and the number of channel taps: $L$ are much smaller than $M$. Also, from Section IVA, we have $Q \ll M N$. Hence, the implementation of the proposed CE scheme is cost effective.

\section{Iterative OTFS Symbol Detection}

By means of the channel responses acquired by the subspace $\mathrm{CE}$, we propose an iterative detection scheme in this section, to retrieve the OTFS data symbols. The basic ideas can be concluded as: 1) we propose a time-domain channel equalizer, which relies on the mathematical LSQR algorithm, to remove the channel distortion on OTFS symbols; 2) by leveraging the equalized OTFS symbols (we call them as the initial OTFS symbol estimates hereafter), the inter-symbol interference (ISI) can be reconstructed, and further removed from observation vector $\mathbf{y}$; 2) after ISI cancellation, the "pure" signal components are accumulated together by using the MRC technique, so as to efficiently extract the multi-path diversity of OTFS channel. 


\section{A. Matrix Form of OTFS Transmission}

Before introducing the proposed detection scheme, we use matrix form to express the OTFS transmission. It can help us investigate the characteristics of channel matrix, and further design the symbol detector.



(a) The observed OTFS signal: $\mathbf{y}=\mathbf{G} \mathbf{x}$.

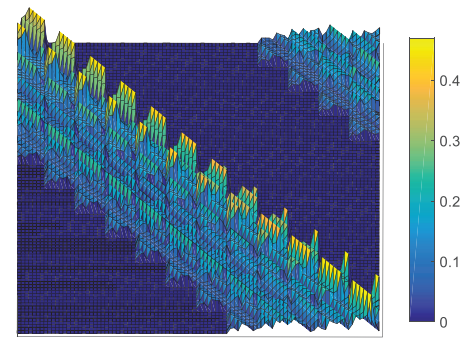

(b) An instance of $\mathbf{G}$.

Fig. 11. The matrix form of OTFS transmission.

Based on (1) and (2), the OTFS transmission can be expressed as (the noise is omitted for simplicity)

$$
\mathbf{y}=\mathbf{G} \mathbf{x}
$$

As shown in Fig. 11, $\mathbf{x} \in \mathbb{C}^{M N \times 1}$ and $\mathbf{y} \in \mathbb{C}^{M N \times 1}$ contains the transmitted and received OTFS symbols, respectively, which are $\mathbf{x}=\left[\mathbf{x}_{0}^{T}, \cdots, \mathbf{x}_{M-1}^{T}\right]^{T} ; \mathbf{y}=\left[\mathbf{y}_{0}^{T}, \cdots, \mathbf{y}_{M-1}^{T}\right]^{T}$. Matrix $\mathbf{G} \in \mathbb{C}^{M N \times M N}$ denotes the OTFS transmission matrix, which is block-banded and follows [30]

$$
\mathbf{G}=\left[\begin{array}{cccc}
\mathbf{G}_{0,0} & \cdots & \mathbf{G}_{L-1,0} & \mathbf{G}_{1,0} \\
\mathbf{G}_{1,1} & \mathbf{G}_{0,1} & \cdots & \mathbf{G}_{2,1} \\
\vdots & \vdots & \ddots & \vdots \\
\cdots & \mathbf{G}_{L-1, M-1} & \cdots & \mathbf{G}_{0, M-1}
\end{array}\right],
$$

where $\mathbf{G}_{\left(l-l^{\prime}\right)_{M}, l} \in \mathbb{C}^{N \times N} ; l, l^{\prime}=0, \cdots, M-1$ is a submatrix. The element in the $v$-th row and $v^{\prime}$-th column of $\mathbf{G}_{\left(l-l^{\prime}\right)_{M}, l}$, is $\mathbf{G}_{\left(l-l^{\prime}\right)_{M}, l}\left(v, v^{\prime}\right)=$ $\Omega\left\{l,\left(l-l^{\prime}\right)_{M}, v-v^{\prime}\right\} ; v, v^{\prime}=0, \cdots, N-1$. From the structure of matrix $\mathbf{G}$, the interference in the OTFS system is generated from two dimensions, namely ISI and inter-Doppler interference (IDI). The ISI is contributed by the submatrices $\mathbf{G}_{1, l}, \cdots, \mathbf{G}_{L-1, l}, l=0, \cdots, M-1$. Meanwhile, the IDI is contributed by the off-diagonal elements of $\mathbf{G}_{\left(l-l^{\prime}\right)_{M}, l}$ in 62.

\section{B. The Proposed Iterative Detector}

The proposed OTFS symbol detector is detailed in Fig. 12 . where the inputs come from the equalized OTFS symbols $\mathbf{x}^{\text {Ini }}$, received data samples $\mathbf{y}$ and DD-domain channel matrix

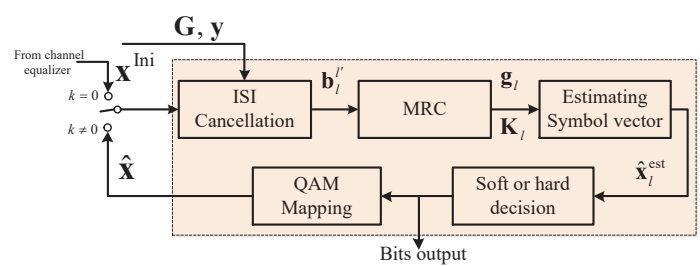

(a) The architecture of the proposed iterative OTFS detector.



(b) Illustrations of ISI cancellation and MRC operation.

Fig. 12. The proposed OTFS symbol detector

G. As illustrated in Fig. 12, the detector mainly consists of ISI cancellation and MRC operation. In the $k$-th iteration, the ISI corresponding to each received OTFS sub-block is reconstructed by the estimated symbols and channel matrix, according to $\sum_{l^{\prime \prime}=0, l^{\prime \prime} \neq l^{\prime}}^{N-1} \mathbf{G}_{l+l^{\prime}, l^{\prime \prime}} \hat{\mathbf{x}}_{l+l^{\prime}-l^{\prime \prime}}$. Then, the observed subvector will be updated through subtracting the resulting ISI: $\mathbf{b}_{l}^{l^{\prime}}=\mathbf{y}_{l+l^{\prime}}-\sum_{l^{\prime \prime}=0, l^{\prime \prime} \neq l^{\prime}}^{N-1} \mathbf{G}_{l+l^{\prime}, l^{\prime \prime}} \hat{\mathbf{x}}_{l+l^{\prime}-l^{\prime \prime}}$, where $\mathbf{b}_{l}^{l^{\prime}}$ can be treated as the "pure" signal of transmit sub-block spreading at the delay direction. After ISI elimination, the MRC is invoked to combine these "pure" signal components, which generates the accumulated signal vector $\mathbf{g}_{l}$ and channel matrix $\mathbf{K}_{l}$. Specifically, the iteration process is summarized in Algorithm 1. The iteration process is described in the following part.

LSQR based time-domain equalization: Recalling (7) in [31], we formulate the time-domain OTFS transmission as the matrix form (omitting noise vector for simplicity)

$$
\mathbf{y}_{\mathrm{TD}}=\mathbf{H}_{\mathrm{TD}} \mathbf{x}_{\mathrm{TD}},
$$

where $\mathbf{y}_{\mathrm{TD}}$ and $\mathbf{x}_{\mathrm{TD}}$ denote the time-domain received and transmitted vectors, respectively; $\mathbf{H}_{\mathrm{TD}} \in \mathbb{C}^{M N \times M N}$ is the time-domain channel matrix constructed by CIR samples. From (63), the time-domain channel equalization is performed by solving the following LS problem

$$
\min _{\hat{\mathbf{x}}_{\mathrm{TD}} \in \mathbb{C}^{M N \times 1}}\left\|\mathbf{H}_{\mathrm{TD}} \hat{\mathbf{x}}_{\mathrm{TD}}-\mathbf{y}_{\mathrm{TD}}\right\|_{2} .
$$

The main features of $\mathbf{H}_{\mathrm{TD}}$ can be summarized: 1) the size of $\mathbf{H}_{\mathrm{TD}}$ can be quite large due to the 2-D block-wise transmission of OTFS; 2) matrix $\mathbf{H}_{\mathrm{TD}}$ is probably ill-conditioned [37]-[39]; 3) it is a sparse matrix. The features motive us to adopt mathematical method, i.e., LSQR, to realize 647. In mathematics, the LSQR [40], was developed to solve largescale, ill-conditioned and sparse LS problems as 64). The method use a recursive strategy to obtain the LS solution with precision similar as the LMMSE method. Meanwhile, by exploiting the sparsity of $\mathbf{H}_{\mathrm{TD}}$, the iteration process needs 


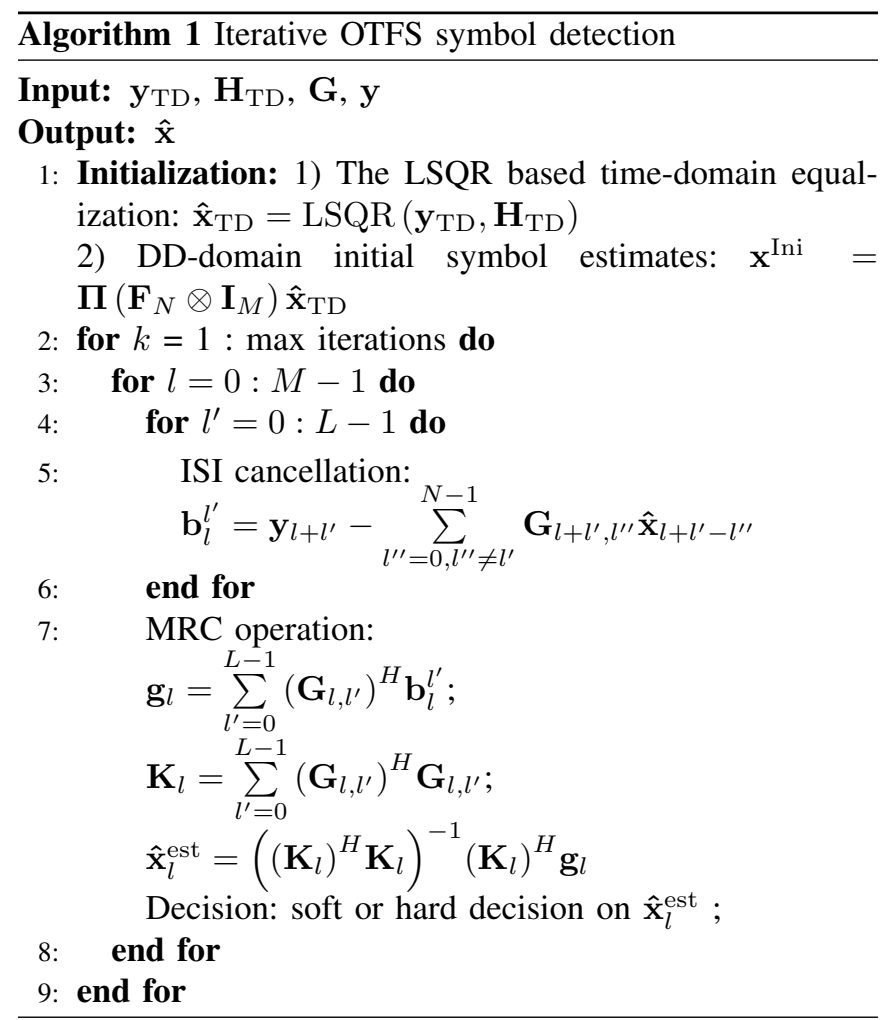

much lower computational overhead. The details of LSQR algorithm can be found in our existing work [8].

DD-domain initial symbol estimates: By transforming the time-domain LSQR estimates to DD domain, we can obtain the accurate initial symbol estimates for the MRC detector. The initial DD-domain symbol estimates are denoted as $\mathbf{x}^{\text {Ini }}$, which follows

$$
\mathbf{x}^{\text {Ini }}=\boldsymbol{\Pi}\left(\mathbf{F}_{N} \otimes \mathbf{I}_{M}\right) \hat{\mathbf{x}}_{\mathrm{TD}}
$$

where $\Pi$ is a permutation matrix [31].

ISI cancellation: In each iteration of MRC scheme, we first propose to calculate $\mathbf{b}_{l}^{l^{\prime}} \in \mathbb{C}^{N \times 1}$, which is also denoted as ISI cancellation. We have

$$
\mathbf{b}_{l}^{l^{\prime}}=\mathbf{y}_{l+l^{\prime}}-\sum_{l^{\prime \prime}=0, l^{\prime \prime} \neq l^{\prime}}^{N-1} \mathbf{G}_{l+l^{\prime}, l^{\prime \prime}} \hat{\mathbf{x}}_{l+l^{\prime}-l^{\prime \prime}},
$$

which is denoted as the channel impaired signal component of $\mathbf{x}_{l}$ in the received vector $\mathbf{y}_{l+l^{\prime}}$ after removing the interference generated by the other transmitted symbol vectors $\hat{\mathbf{x}}_{l+l^{\prime}-l^{\prime \prime}}$ for $l^{\prime}-l^{\prime \prime} \neq 0[32]$.

MRC operation: From 66, we have

$$
\left\{\begin{array}{c}
\left(\mathbf{G}_{l, 0}\right)^{H} \mathbf{b}_{l}^{0}=\left(\mathbf{G}_{l, 0}\right)^{H} \mathbf{G}_{l, 0} \hat{\mathbf{x}}_{l}+\mathbf{w}_{l} \\
\vdots \\
\left(\mathbf{G}_{l, L-1}\right)^{H} \mathbf{b}_{l}^{L-1}=\left(\mathbf{G}_{l, L-1}\right)^{H} \mathbf{G}_{l, L-1} \hat{\mathbf{x}}_{l}+\mathbf{w}_{l+L-1}
\end{array}\right.
$$

By combing the whole equations in (67), we have

$$
\sum_{l^{\prime}=0}^{L-1}\left(\mathbf{G}_{l, l^{\prime}}\right)^{H} \mathbf{b}_{l}^{l^{\prime}}=\left(\sum_{l^{\prime}=0}^{L-1}\left(\mathbf{G}_{l, l^{\prime}}\right)^{H} \mathbf{G}_{l, l^{\prime}}\right) \hat{\mathbf{x}}_{l}+\tilde{\mathbf{w}}_{l}
$$

where $\tilde{\mathbf{w}}_{l}=\sum_{l^{\prime}=0}^{L-1} \tilde{\mathbf{w}}_{l+l^{\prime}}$. Defining

$$
\mathbf{g}_{l}=\sum_{l^{\prime}=0}^{L-1}\left(\mathbf{G}_{l, l^{\prime}}\right)^{H} \mathbf{b}_{l}^{l^{\prime}} ; \mathbf{K}_{l}=\sum_{l^{\prime}=0}^{L-1}\left(\mathbf{G}_{l, l^{\prime}}\right)^{H} \mathbf{G}_{l, l^{\prime}},
$$

the estimation of $\hat{\mathbf{x}}_{l}$ is performed as

$$
\hat{\mathbf{x}}_{l}^{\mathrm{est}}=\left(\left(\mathbf{K}_{l}\right)^{H} \mathbf{K}_{l}\right)^{-1}\left(\mathbf{K}_{l}\right)^{H} \mathbf{g}_{l} \text {. }
$$

At the end, the hard or soft decision strategy will be adopted for detecting $\hat{\mathbf{x}}_{l}^{\text {est }}$ as data symbols.

\section{Simulations AND Discussions}

In this section, the performance of $\mathrm{CE}$ and data detection is evaluated for the OTFS system, where the carrier frequency $f_{c}$ $=5.9 \mathrm{GHz}$ and subcarrier spacing $\Delta f=15 \mathrm{KHz}$. Two OTFS transmission frames with grid sizes of $(M, N)=32 \times 32$ and $32 \times 128$ are considered. A recursive systematic convolutional code with the generator $[1,5 / 7]_{8}$ and coding rate of $1 / 2$ is employed. The data bits, out of the channel encoder, are mapped to the 16-QAM, 64-QAM and 256-QAM symbols 5 . The pilots, generated by the Zadoff Chu sequences, are inserted into the OTFS transmission frame as illustrated in Fig. 9. The averaged pilot power is denoted as $T \mathrm{dBW}$. The extended vehicular A model, which is specified by $3 \mathrm{GPP}$ for high-speed mobile scenarios [33], is adopted. In the channel simulator, each path is assumed as independently and identically distributed random variables correlated in time according to the classical Clarke model with maximum Doppler frequency $f_{d}$ [41]. In this paper, we simulate some scenarios with $\tilde{f}_{d}=f_{d} / \Delta f$ ranging from $5 \%$ to $30 \%$, corresponding the velocities from $137 \mathrm{~km} / \mathrm{h}$ to $685 \mathrm{~km} / \mathrm{h}$. To acquire validated results, as least $1 \times 10^{5}$ OTFS transmission blocks are simulated in each case.

\section{A. CE and Demodulation Performance in $(32,32)$ OTFS System}

We first investigate the $\mathrm{CE}$ and demodulation performance in $(32,32)$ OTFS System. In the system, we consider $N_{\mathrm{P}}=$ $N / 2$ and $M_{\mathrm{P}}=1,2,3$ in the CIPP, corresponding to the transmission efficiency $\eta=68.75 \%, 75 \%$ and $81.25 \%$, respectively. Note that when $N_{\mathrm{P}}=N / 2$, the shape of CIPP in Fig. 9 is changed, where the pilot symbols occupies the whole grids in the Doppler direction. Additionally, two stateof-the-art schemes, i.e., the SI-based CE [25] and CS-based CE [28], are included for comparison. The pilot overhead ratio is set as $50 \%$ for both of them, corresponding to $\eta=$ $50 \%$.

Fig. 13 compares the equivalent channel responses (ECRs) tracking curves of the $l_{0}^{\prime}$-th path and $v_{0}^{\prime}$-th Doppler frequency. The $\tilde{f}_{d}$ is set as $20 \%$ to demonstrate significant variation of the ECRs $\Omega\left\{l, l_{0}^{\prime}, v_{0}^{\prime}\right\}$ with respect to group index $l$. The estimated ECRs through our method, denoted as the red line, highly coincide with the ideal response. However,

\footnotetext{
${ }^{5}$ Note that the proposed CE and symbol detection schemes are not only applicable to QAM constellation, but also can be used to other constellations, e.g., pulse amplitude modulation or phase shift keying.
} 


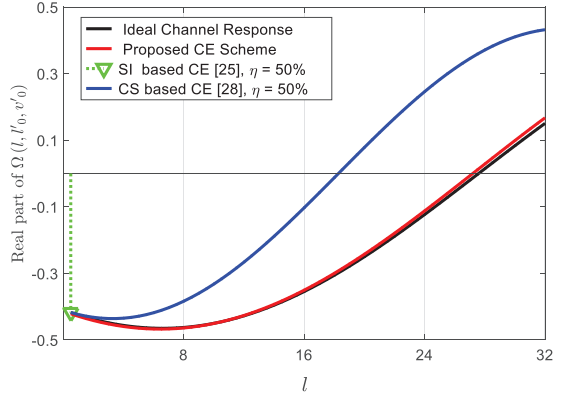

Fig. 13. The ECRs tracking curves of the $l_{0}^{\prime}$-th path and $v_{0}^{\prime}$-th Doppler frequency. The maximum Doppler frequency normalized to subcarrier spacing $f_{d}=20 \%$, corresponding to a velocity of $550 \mathrm{~km} / \mathrm{h}$.

the SI-based CE approach [25], can only obtain a singlepoint response during the whole groups. The CS-based CE method [28] can estimate the first few channel responses while introduces obvious estimation error as $l$ increases.



Fig. 14. The MSE versus SNR in $(32,32)$ OTFS system, where the pilot power $T$ is $0 \mathrm{dBW}$. In the Clarke channel, $\tilde{f}_{d}=20 \%$, corresponding to velocities of $550 \mathrm{~km} / \mathrm{h}$.

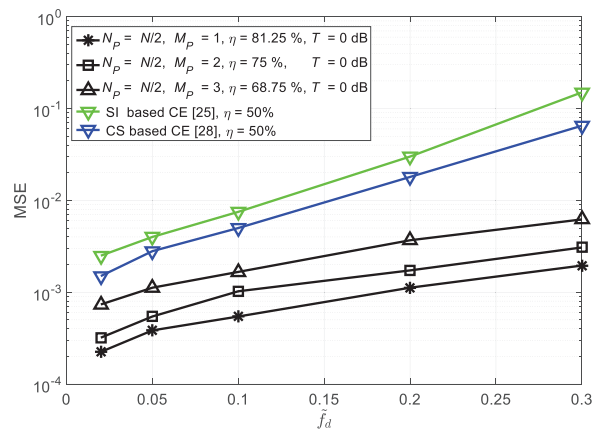

Fig. 15. The MSE floor comparison of $\tilde{f}_{d}$ in $(32,32)$ OTFS system. The pilot power $T=0 \mathrm{dBW}$ and the noise variance $\sigma^{2}=0.01$.

Fig. 14 demonstrates the MSE performance comparison against the SNR when $\tilde{f}_{d}=20 \%$. The SI based CE approach [25] is to obtain the single-point ECR during one symbol duration. Therefore, to evaluate the average MSE, the estimated ECRs are regarded as constant within the interval $l \in[0, M-1]$. From Fig. 14 the proposed CE scheme significantly outperforms the CE schemes respectively developed in [25] and [28]. As the SI-based CE scheme [25] ignores the variation of $\Omega\left\{l, l^{\prime}, v^{\prime}\right\}$ with respect to group index $l$, an MSE floor exists when SNR $\geq 20 \mathrm{~dB}$. For the CS-based CE scheme [28], it outperforms the SI-based CE but still introduces an MSE floor. The comparison of the MSE floor against $\tilde{f}_{d}$ is presented in Fig. 15 , where noise variance is 0.01. For the proposed CE scheme, given the threshold MSE less than $2 \times 10^{-3}$, the $\tilde{f}_{d}$ thresholds for $\eta=68.75 \%, 75 \%$ and $81.25 \%$ cases are $0.3,0.23$ and 0.12 , respectively. In contrast, for the considered $\tilde{f}_{d}$ regime, the MSE values of other CE schemes cannot reach the level.

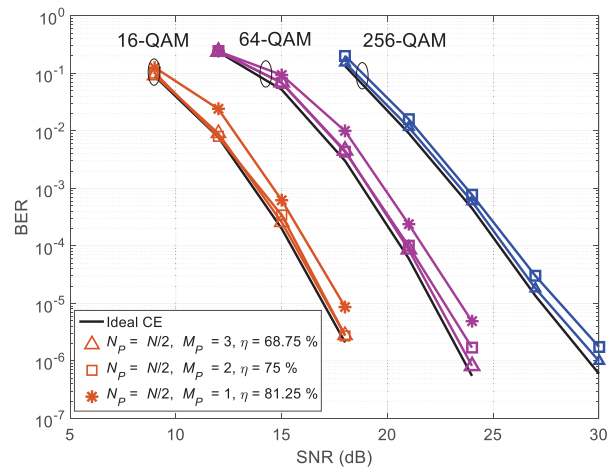

Fig. 16. The BER comparison of SNR in $(32,32)$ OTFS system when $\tilde{f}_{d}$ $=5 \%$. The pilot power $T=0 \mathrm{dBW}$.

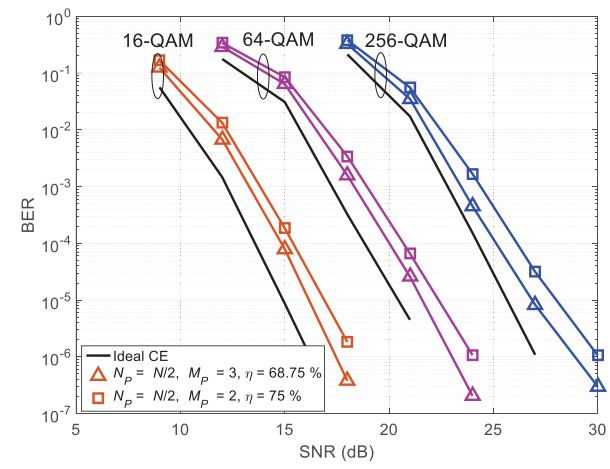

Fig. 17. The BER comparison of SNR in $(32,32)$ OTFS system when $\tilde{f}_{d}$ $=20 \%$. The pilot power $T=0 \mathrm{dBW}$.

Figs. 16 and 17 compare the BER versus SNR for different modulation schemes in velocities of $137 \mathrm{~km} / \mathrm{h}$ and $550 \mathrm{~km} / \mathrm{h}$, respectively. In Fig. 16 , for BER $=1 \times 10^{-5}$, the required demodulation SNRs with ideal CE for 16-QAM, 64-QAM and 256-QAM signaling are about $17 \mathrm{~dB}, 22.2 \mathrm{~dB}$ and $27.3 \mathrm{~dB}$, respectively. The SNR loss under three CE cases, corresponding to $\eta=68.75 \%, 75 \%$ and $81.25 \%$, is less than 1.3 dB. From Fig. 17 , when $\tilde{f}_{d}=20 \%$, the SNR loss under the CE cases of $\eta=68.75 \%$ and $75 \%$ is less than $2.5 \mathrm{~dB}$.

In practice, increasing the pilot power is an alternative method to improve the CE accuracy in high-mobility environments. By doing so, the transmission power for the whole OTFS block is magnified by

$$
\Upsilon=10 \lg \left(\frac{\text { Pis } \times 10^{T / 10}+\text { Das }}{\text { Pis }+ \text { Das }}\right) \mathrm{dB} .
$$

Figs. 18 and 19 demonstrate the BER and peak-to-average power ratio (PAPR) performance when adopting different 


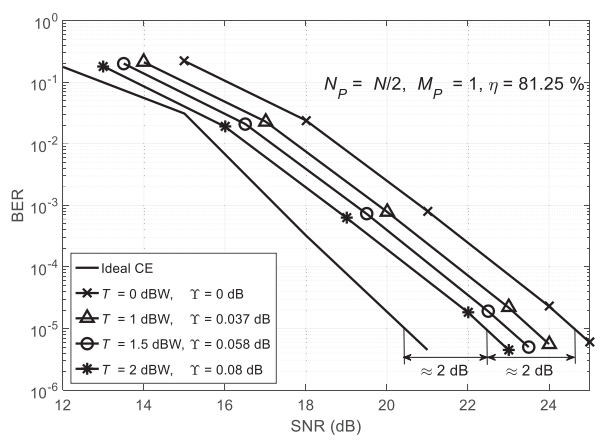

Fig. 18. The BER performance for different pilot power $T$ in $(32,32)$ OTFS system with 64-QAM signaling, where $\tilde{f}_{d}=20 \%$. In the CIPP, $N_{\mathrm{P}}=N / 2$ and $M_{\mathrm{P}}=1$, related to $\eta=81.75 \%$.

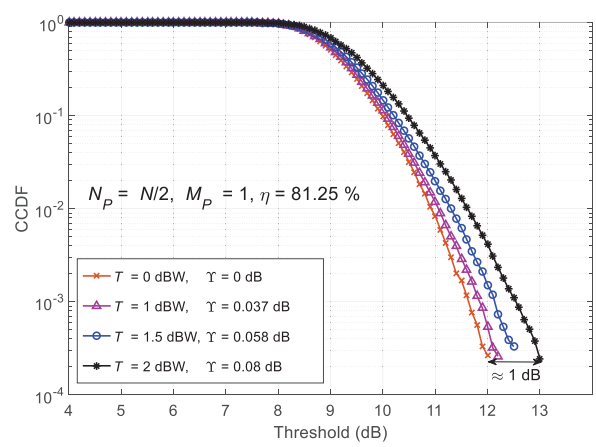

Fig. 19. The PAPR performance in $(32,32)$ OTFS system with 64-QAM signaling and different pilot power $T$.

pilot power. In Fig. 18, the black line without marker denotes the BER performance of 64-QAM signaling with ideal CE. Without pilot power improvement, the SNR loss under case " $T=0 \mathrm{dBW}, \Upsilon=0 \mathrm{~dB}$ " is about $4 \mathrm{~dB}$. When enhancing the pilot power by 1 to $2 \mathrm{~dB}$, the MSE curve of case " $T=0 \mathrm{dBW}, \Upsilon=0 \mathrm{~dB}$ " in Fig. 14 will move toward the left by 1 to $2 \mathrm{~dB}$. Thus, the SNR gaps in Fig. 18 can be directly reduced by 1 to $2 \mathrm{~dB}$. Since the pilot overhead is relatively small, the increased power $\Upsilon$ for the whole OTFS block in (71) could be neglected. On the other hand, the PAPR performance will be deteriorated with the pilot power enhancement, which is illustrated in Fig. 19. As we can see, given a complementary cumulative distribution function (CCDF) level of $3 \times 10^{-4}$, the threshold for case " $T=2 \mathrm{dBW}, \Upsilon=0.08 \mathrm{~dB}$ " will be magnified by $1 \mathrm{~dB}$, in contrast to case " $T=0 \mathrm{dBW}, \Upsilon=0 \mathrm{~dB}$ ". Hence, in the practical OTFS system, there should be a tradeoff between the BER performance, pilot overhead (transmission efficiency), pilot power and PAPR when facing the high mobilities.

\section{B. CE and Demodulation Performance in $(32,128)$ OTFS System}

We also investigate the proposed $\mathrm{CE}$ and data detection schemes in $(32,128)$ OTFS System, where the maximum Doppler frequency normalized to the subcarrier spacing $\tilde{f}_{d}=$ $10 \%$, corresponding to a moderate velocity of $275 \mathrm{~km} / \mathrm{h}$. We consider two pilot configurations, i.e., "Pattern I: $N_{\mathrm{P}}=N / 8$, $M_{\mathrm{P}}=2$ " and "Pattern II: $N_{\mathrm{P}}=N / 8, M_{\mathrm{P}}=1$ ".



Fig. 20. The MSE versus SNR in $(32,128)$ OTFS system, where the pilot power $T$ is $0 \mathrm{dBW}$. In the Clarke channel, $\tilde{f}_{d}=10 \%$, corresponding to a velocity of $275 \mathrm{~km} / \mathrm{h}$.



Fig. 21. The error surface of the proposed and SI-based CE [25] schemes, where the noise variance $\sigma^{2}=0.01$. In the CIPP, pilot configuration is $N_{\mathrm{P}}=N / 8, M_{\mathrm{P}}=1$ and $N_{\text {data }}=N / 16$, which yields $\eta=81.75 \%$.

Accordingly, the extra grids in Fig. 9 with the number of $2 N_{\text {data }}\left(M_{\mathrm{P}} M_{\mathrm{GI}}\right)$ are used to place QAM symbols, achieving an improved transmission efficiency.

Fig. 20 compares the MSE versus SNR for patterns I and II. The MSE lower bound is calculated from (59) and 60) by considering $N_{\text {data }}=0$. When SNR $<25 \mathrm{~dB}$, the extra data symbols hardly affect the $\mathrm{CE}$ accuracy. At a high SNR regime, such as $\mathrm{SNR} \geq 25 \mathrm{~dB}$, the interference generated by data symbols, gradually deteriorates the CE accuracy. To achieve the satisfactory $\mathrm{CE}$ performance and transmission efficiency simultaneously, we choose $N_{\mathrm{P}}=N / 8, M_{\mathrm{P}}=2, N_{\text {data }}=$ $N / 16$ for Pattern I, and $N_{\mathrm{P}}=N / 8, M_{\mathrm{P}}=1, N_{\text {data }}=N / 16$ for Pattern II.

Fig. 21 demonstrates the CE error surface of the $l_{0}^{\prime}$-th path under the SI-based CE method and ours, where

$$
\varepsilon\left(l, l_{0}^{\prime}, v^{\prime}\right)=\Omega\left(l, l_{0}^{\prime}, v^{\prime}\right)-\hat{\Omega}\left(l, l_{0}^{\prime}, v^{\prime}\right) .
$$

We can see that the proposed CE method significantly outperforms the SI-based CE scheme [25]. Benefiting from the precise channel estimates, the values of $\left\|\varepsilon\left(l, l_{0}^{\prime}, v^{\prime}\right)\right\|^{2}$ are mostly lower than $1 \times 10^{-5}$ in our scheme.

Fig. 22 compares the BER versus SNR in $(32,128)$ OTFS system, where the pilot power $T$ is set as $2 \mathrm{dBW}$. For 256QAM signaling, the SNR losses under cases $N_{\mathrm{P}}=N / 8$, $M_{\mathrm{P}}=2, N_{\text {data }}=N / 16$ and $N_{\mathrm{P}}=N / 8, M_{\mathrm{P}}=1, N_{\text {data }}=$ $N / 16$ are about $0.9 \mathrm{~dB}$ and $2.1 \mathrm{~dB}$, respectively, at BER = $2 \times 10^{-5}$. For 64-QAM signaling, the maximum SNR loss caused by $\mathrm{CE}$ error is about $1.3 \mathrm{~dB}$. 


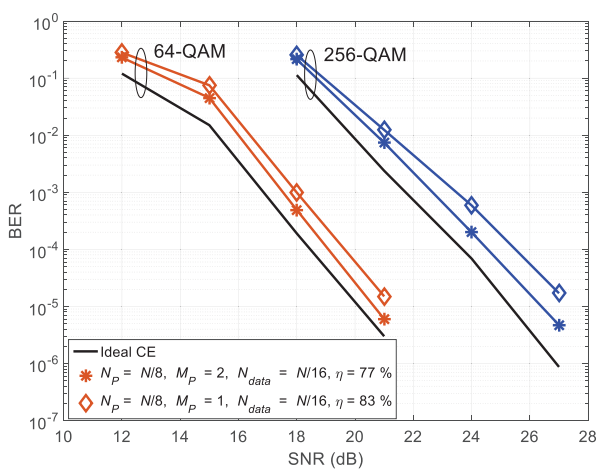

Fig. 22. The BER comparison of SNR in $(32,128)$ OTFS system when $\tilde{f}_{d}=10 \%$. The pilot power $T=2 \mathrm{dBW}$.

\section{Conclusions}

In this paper, we studied the orthogonal time frequency space (OTFS) signal transmission over the continuousDoppler-spread channel with the focus on the channel estimation (CE) and data detection. Specifically, through analyzing the equivalent channel responses (ECRs) in OTFS system, we found that the ECRs change significantly within one symbol block in high-mobility environments. To precisely track the variations of ECRs, we constructed a low-dimensional subspace to characterize the OTFS channel, and further develop the subspace CE scheme. Based on the estimated ECRs, an iterative maximal ratio combing detector was developed by introducing the liner-complexity interference eliminator, as well as least-squares QR decomposition based channel equalizer. Simulation results verified that, in high-mobility environments, the developed OTFS receiver can ideally demodulate 256-ary quadrature amplitude modulation symbols, and the $\mathrm{CE}$ error introduced demodulation performance loss is less than $3 \mathrm{~dB}$.

\section{REFERENCES}

[1] 3GPP, Study on enhancement of 3GPP Support for $5 G$ V2X Services, 3GPP Release 15, TR 22.886 V15.1.0 Std., 2017.

[2] P. Wang, B. Di, H. Zhang, K. Bian, and L. Song, "Cellular V2X communications in unlicensed spectrum: Harmonious coexistence with VANET in $5 \mathrm{G}$ systems," IEEE Trans. Wireless Commun., vol. 17, no. 8, pp. 5212-5224, Aug. 2018.

[3] C. X. Wang, A. Ghazal, B. Ai, Y. Liu, and P. Fan, "Channel measurements and models for high-speed train communication systems: A survey," IEEE Commun. Surveys \& Tutorials, vol. 18, no. 2, pp. 974987, Dec. 2015.

[4] C. Zhang and P. Fan, "Providing services for the high-speed train and local users in the same OFDMA system: Resource allocation in the downlink," IEEE Trans. Wireless Commun., vol. 15, no. 2, pp. 10181030, Feb. 2016.

[5] M. Mozaffari, W. Saad, M. Bennis, and M. Debbah, "Unmanned aerial vehicle with underlaid device-to-device communications: Performance and tradeoffs," IEEE Trans. Wireless Commun., vol. 15, no. 6, pp. 39493963, June 2016.

[6] L. Yang and W. Zhang, "Beam tracking and optimization for UAV communications," IEEE Trans. Wireless Commun., vol. 18, no. 11, pp. 5367-5379, Nov. 2019.

[7] G. Liu, L. Zeng, H. Li, L. Xu, and Z. Wang, "Adaptive interpolation for pilot-aided channel estimator in OFDM system," IEEE Trans. Broadcast., vol. 60, no. 3, pp. 486-498, June 2014.

[8] H. Qu, G. Liu, Y. Wang, Q. Chen, C. Yi, and J. Peng, "A timedomain approach to channel estimation and equalization for the SCFDM system," IEEE Trans. Broadcast., vol. 65, no. 4, pp. 713-726, Dec. 2019.
[9] H. Senol, E. Panayirci, and H. V. Poor, "Nondata-aided joint channel estimation and equalization for OFDM systems in very rapidly varying mobile channels," IEEE Trans. Signal Process., vol. 60, no. 8, pp. 4236-4253, Nov. 2012.

[10] L. Zhang, A. Ijaz, P. Xiao, M. M. Molu, and R. Tafazolli, "Filtered OFDM systems, algorithms, and performance analysis for $5 \mathrm{G}$ and beyond," IEEE Trans. Commun., vol. 66, no. 3, pp. 1205-1218, Mar. 2017.

[11] L. Zhang, P. Xiao, A. Zafar, A. U. Quddus, and R. Tafazolli, "FBMC system: an insight into doubly dispersive channel impact," IEEE Trans. Veh. Technol., vol. 66, no. 5, pp. 3942-3956, May 2017.

[12] X. Zhang, L. Zhang, P. Xiao, D. Ma, J. Wei, and Y. Xin, "Mixed numerologies interference analysis and inter-numerology interference cancellation for windowed OFDM systems," IEEE Trans. Veh. Technol., vol. 67, no. 8, pp. 7047-7061, Aug. 2018.

[13] G. Surabhi, R. M. Augustine, and A. Chockalingam, "Peak-to-average power ratio of OTFS modulation," IEEE Commun. Lett., vol. 23, no. 6, pp. 999-1002, June 2019.

[14] R. Hadani, S. Rakib, M. Tsatsanis, A. Monk, A. J. Goldsmith, A. F. Molisch, and R. Calderbank, "Orthogonal time frequency space modulation," in Proc. IEEE Wireless Communications and Networking Conference (WCNC), Mar. 2017.

[15] R. M. Augustine, G. Surabhi, and A. Chockalingam, "Space-time coded OTFS modulation in high-Doppler channels," in Proc. IEEE 89th Vehicular Technology Conference (VTC2019-Spring), May 2019.

[16] R. Hadani and A. Monk, "OTFS: A new generation of modulation addressing the challenges of 5G," arXiv preprint: 1802.02623, 2018.

[17] A. Farhang, A. Rezazadehreyhani, L. E. Doyle, and B. FarhangBoroujeny, "Low complexity modem structure for OFDM-based orthogonal time frequency space modulation," IEEE Wireless Commun. Lett., vol. 7, no. 3, pp. 344-347, Nov. 2017.

[18] A. Rezazadehreyhani, A. Farhang, M. Ji, R. R. Chen, and B. FarhangBoroujeny, "Analysis of discrete-time MIMO OFDM-based orthogonal time frequency space modulation," in Proc. IEEE International Conference on Communications (ICC), May 2018.

[19] P. Raviteja, P. K. T., H. Yi, and V. Emanuele, "Interference cancellation and iterative detection for orthogonal time frequency space modulation," IEEE Trans. Wireless Commun., vol. 17, no. 10, pp. 6501-6515, Aug. 2018.

[20] G. Surabhi, R. M. Augustine, and A. Chockalingam, "On the diversity of uncoded OTFS modulation in doubly-dispersive channels," IEEE Trans. Wireless Commun., vol. 18, no. 6, pp. 3049-3063, June 2019.

[21] F. Long, K. Niu, C. Dong, and J. Lin, "Low complexity iterative LMMSE-PIC equalizer for OTFS," in Proc. IEEE International Conference on Communications (ICC), May 2019.

[22] W. Yuan, Z. Wei, J. Yuan, and D. W. K. Ng, "A simple variational bayes detector for orthogonal time frequency space (OTFS) modulation," IEEE Trans. Veh. Technol., vol. 69, no. 7, pp. 7976-7980, July 2020.

[23] C. X. Wang, A. Ghazal, B. Ai, Y. Liu, and P. Fan, "Channel measurements and models for high-speed train communication systems: A survey." IEEE Commun. Surv. Tut., vol. 18, no. 2, pp. 974-987, Dec. 2016.

[24] R. Hadani, S. Rakib, A. Molisch, C. Ibars, A. Monk, M. Tsatsanis, J. Delfeld, A. Goldsmith, and R. Calderbank, "Orthogonal time frequency space (otfs) modulation for millimeter-wave communications systems," in Proc. IEEE MTT-S International Microwave Symposium (IMS), Oct. 2017.

[25] P. Raviteja, K. T. Phan, and Y. Hong, "Embedded pilot-aided channel estimation for OTFS in delay-Doppler channels," IEEE Trans. Veh. Technol., vol. 68, no. 5, pp. 4906-4917, May 2019.

[26] M. K. Ramachandran and A. Chockalingam, "MIMO-OTFS in highDoppler fading channels: signal detection and channel estimation," in Proc. IEEE Global Communications Conference (GLOBECOM), Dec. 2018.

[27] K. Murali and A. Chockalingam, "On OTFS modulation for highDoppler fading channels," in Proc. IEEE Information Theory and Applications Workshop (ITA), Oct. 2018.

[28] W. Shen, L. Dai, J.-p. An, P. Fan, and R. W. Heath, "Channel estimation for orthogonal time frequency space (OTFS) massive MIMO," IEEE Trans. Signal Process., vol. 67, no. 16, pp. 4204-4217, Aug. 2019.

[29] 3GPP, Study on LTE-based V2X Services, Rel. 14, TR 36.885 (V14.0.0) Std., 2016.

[30] H. Qu, G. Liu, L. Zhang, S. Wen, and M. A. Imran, "Low-complexity symbol detection and interference cancellation for OTFS system," IEEE Trans. Commun., 2020, Early Access. 
[31] P. Raviteja, Y. Hong, E. Viterbo, and E. Biglieri, "Practical pulseshaping waveforms for reduced-cyclic-prefix OTFS," IEEE Trans. Veh. Technol., vol. 68, no. 1, pp. 957-961, Jan. 2019.

[32] T. Thaj and E. Viterbo, "Low complexity iterative rake decision feedback equalizer for OTFS modulation," in Proc. IEEE Wireless Communications and Networking Conference (WCNC), May 2020.

[33] 3GPP, Base Station (BS) conformance testing, Rel. 15, TS 36.141 (V15.1.0) Std., 2017.

[34] F. Mikael, et al., "Fifth-generation technologies for the connected car: Capable systems for vehicle-to-anything communications," IEEE Veh. Technol. Magazine, vol. 13, no. 3, pp. 28-38, Sept. 2018

[35] R. H. Clarke, "A statistical theory of mobile-radio reception," Bell Syst. Tech., pp. 957-1000, July 1968.

[36] Y. R. Zheng and C. Xiao, "Improved models for the generation of multiple uncorrelated rayleigh fading waveforms," IEEE Commun. Lett., vol. 6, no. 6, pp. 256-258, Aug. 2011.

[37] J. Tong, Q. Guo, S. Tong, J. Xi, and Y. Yu, "Condition numberconstrained matrix approximation with applications to signal estimation in communication systems," IEEE Signal Process. Lett., vol. 21, no. 8, pp. 990-993, Aug. 2014.

[38] Y. Rong, S. A. Vorobyov, and A. B. Gershman, "Robust linear receivers for multiaccess space-time block-coded mimo systems: a probabilistically constrained approach," IEEE J. Sel. Areas Commun., vol. 24, no. 8 , pp. $1560-1570$, Aug. 2006.

[39] O. Ledoit and M. Wolf, "A well-conditioned estimator for largedimensional covariance matrices," J. Multivar. Anal., vol. 88, no. 2, pp. 365-411, 2004.

[40] C. C. Paige and M. A. Saunders, "LSQR: An algorithm for sparse linear equations and sparse least squares," ACM Trans. Mathemat. Software, vol. 8, no. 1, pp. 43-71, 1982.

[41] C. Xiao, Y. R. Zheng, and N. C. Beaulieu, "Second-order statistical properties of the WSS Jakes' fading channel simulator," IEEE Trans. Commun., vol. 50, no. 6, pp. 888-891, June 2002.

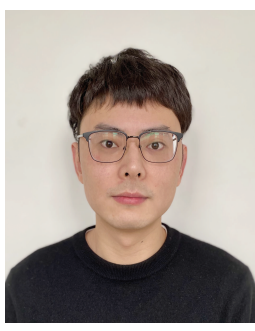

Huiyang Qu received the B.Sc. degree from the University of Electronic Science and Technology of China (UESTC), Chengdu, China in 2015. He is currently working toward the Ph.D. degree at UESTC. His research interests include channel estimation and equalization, $5 \mathrm{G}$ and beyond waveform design and mmWave communication system.

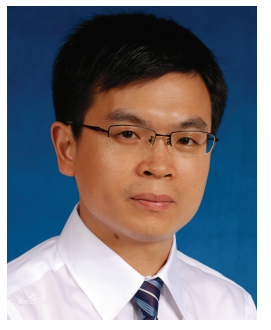

Guanghui Liu (Senior member, IEEE) received the M.Sc. and Ph.D. degrees in electronic engineering from the University of Electronic Science and Technology of China (UESTC), Chengdu, China, in 2002 and 2005, respectively. In 2005, he joined Samsung Electronics, South Korea, as a Senior Engineer. In 2009, he became an Associate Professor with the School of Electronics Engineering, UESTC, where he has been a Full Professor since 2014 and is currently with the School of Information and Communication Engineering. His general research interests include digital signal processing and telecommunications, with emphasis on digital video processing and transmission, 5G waveform design, and OFDM techniques. He has published tens of papers in refereed journals or conferences, and received over 60 patents (6 U.S. granted patents) in the above areas. He was a recipient of the Natural Science Award and the Science and Technology Progress Award, both from Ministry of Education of China in 2015. He served as the Publication Chair of IEEE ISPACS-2010 and IEEE VCIP-2016.

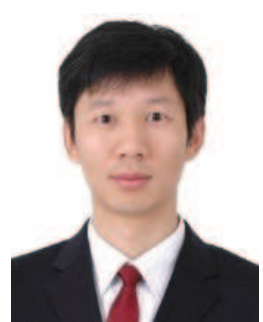

Lei Zhang (Senior member, IEEE) is a Senior Lecturer at the University of Glasgow, U.K. $\mathrm{He}$ received his $\mathrm{Ph} . \mathrm{D}$. from the University of Sheffield, U.K. His research interests include wireless communication systems and networks, blockchain technology, data privacy and security, radio access network slicing (RAN slicing), Internet of Things (IoT), multi-antenna signal processing, MIMO systems, etc. He has 19 patents granted/filed in more than 30 countries/regions including US/UK/EU/China/Japan etc. Dr Zhang has published 2 books and 100+ peer-reviewed papers. He received IEEE Communication Society TAOS Best Paper Award 2019. He is a Technical Committee Chair of 5th International conference on UK-China Emerging Technologies (UCET) 2020. He was the Publication and Registration Chair of IEEE Sensor Array and Multichannel (SAM) 2018, Co-chair of Cyber-C Blockchain workshop 2019. He is an associate editor of IEEE Internet of Things (IoT) Journal, IEEE Wireless Communications Letters and Digital Communications and Networks. His research is covered by media widely including BBC.

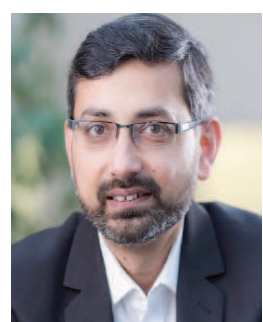

Muhammad Ali Imran (Senior Member, IEEE) is currently a Professor of wireless communication systems at the University of Glasgow, where he heads the Communications, Sensing and Imaging (CSI) research group, and is also the Dean of the University of Glasgow, UESTC. He is also an Affiliate Professor at the University of Oklahoma, USA, and a Visiting Professor at the 5G Innovation Centre, University of Surrey, U.K. He has over 20 years of combined academic and industry experience with several leading roles in multimillion pounds funded projects. He has authored or co-authored over 400 journals and conference publications, was an editor of five books, and an author of more than 20 book chapters, and has filed 15 patents. He has also successfully supervised over 40 postgraduate students at doctoral level. His research interests are in self-organized networks, wireless networked control systems, and wireless sensor systems. He has been a consultant to international projects and local companies in the area of self-organized networks. He has been interviewed by BBC, Scottish television, and many radio channels on the topic of $5 \mathrm{G}$ technology. He is a fellow of IET and a Senior Fellow of HEA.



Shan Wen (Student member, IEEE) received the B.Sc. degree from the University of Electronic Science and Technology of China (UESTC), Chengdu, China, in 2017, where $\mathrm{He}$ is currently working toward the Ph.D. degree. His research interests include faster-than-Nyquist signaling, channel estimation and iterative equalization, MIMO-FTN system. 